\title{
Hybrid inorganic-organic fluorescent silica nanoparticles-influence of dye binding modes on dye leaching
}

\author{
Nadja Klippel ${ }^{1}$ Gregor Jung $\mathbb{D}^{2} \cdot$ Guido Kickelbick $\mathbb{B}^{1}$
}

Received: 24 March 2021 / Accepted: 16 June 2021

(c) The Author(s) 2021

\begin{abstract}
Silica nanoparticles with embedded fluorescent dyes represent an important class of markers for example in biological imaging. We systematically studied the various incorporation mechanisms of fluorescent xanthene dyes in $30-40 \mathrm{~nm}$ silica nanoparticles. An important parameter was the interaction of the dye with the matrix material, either by weak electrostatic or strong covalent interactions, which also has implications on the stability of fluorescence and brightness of the dyes. Factors that can influence leaching of dyes such as the position of the dyes in particles and the intensity of the particle-dye interaction were investigated by using the solvatochromic effect of xanthene dyes and by stationary fluorescence anisotropy measurements. We compared uranine and rhodamine B, which were physically embedded, with modified fluorescein isothiocyanate and rhodamine B isothiocyanate, which were covalently bound to the silica matrix within a usual Stöber synthesis. Systematic leaching studies of time spans up to 4 days revealed that covalent bonding of dyes like fluorescein isothiocyanate or rhodamine B isothiocyanate is necessary for fluorescence stability, since dyes bound by physical interaction tend to leach out of porous silica networks. Coverage of silica particles with hydrophobic protection layers of alkyltrialkoxysilanes or hydrophilic polyethylene glycol (PEG) groups resulted in a better retention of physisorbed dyes and provides the possibility to adapt the particles to the polarity of the medium. Best results were archived with PEG groups, but even small trimethylsilyl (TMS) groups already reduce leaching.
\end{abstract}

\section{Graphical Abstract}

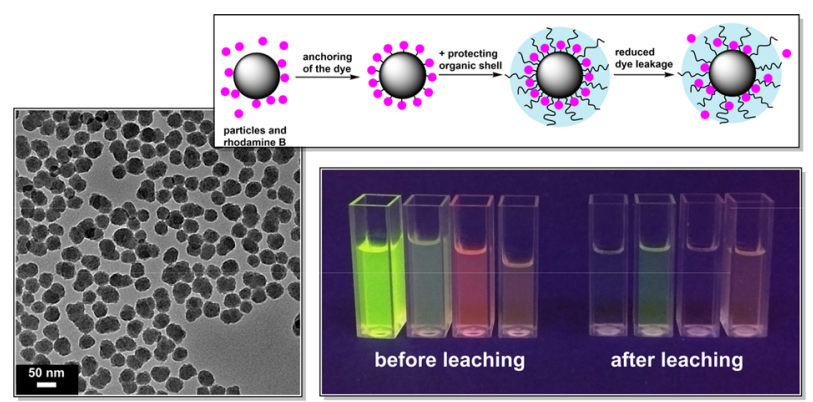

Keywords Silica nanoparticles $\cdot$ Fluorescent dyes $\cdot$ Dye leaching $\cdot$ Nanoparticle morphology

Supplementary information The online version contains

supplementary material available at https://doi.org/10.1007/s10971021-05578-y.

Guido Kickelbick

guido.kickelbick@uni-saarland.de

1 Saarland University, Inorganic Solid-State Chemistry, Campus C4 1, 66123 Saarbrücken, Germany
2 Saarland University, BioPhysical Chemistry, Campus B2 2, 66123 Saarbrücken, Germany 


\section{Highlights}

- Silica nanoparticles prepared by the sol-gel process are an important platform for the formation of fluorescent nanoparticles.

- Fluorescent dyes can be incorporated into the particles by either physical embedment or covalent attachment to the silica network.

- Dye leaching behavior can be controlled depending on the interaction mechanisms with the silica network.

- The leaching of only physically entrapped dyes can be dramatically reduced if a surface-functionalization with alkoxy silanes is carried out.

\section{Introduction}

Fluorescent particles are important materials for a broad variety of different applications such as imaging agents in biological applications [1-3], organic dye based LED materials [4], as markers for understanding migration phenomena of particles in polymers [5]. Functionalized silica particles are particularly interesting in such applications due to their straightforward and well-established synthetic procedures such as the sol-gel based Stöber process [6], reverse microemulsions [7], or with approaches like the so called Hartlen-synthesis [8] or other amino acid based synthesis [9]. Compared to fluorescent quantum dots, which can also be used as fluorescent particles, the hybrid particles show advantages with respect to optical stability, quantum yields, toxicity, emission independent size control, and surfacefunctionalization. This makes hybrid particles an excellent alternative to quantum dots.

In principle there are two chemical approaches to incorporate organic dyes into silica nanoparticles. A physically incorporation in which the dye interacts with the porous silica network via non-covalent interactions like hydrogen bonds, van der Waals forces, or ionic interactions. For this synthetic approach it is necessary that the dye matches parameters such as polarity during the particle formation. The drawback of this encapsulation method is the risk of dye leakage [10, 11], caused by the rather weak interaction between dye and particle as well as the porous nature of the particles. Modification of the dye molecules or the particle surface with functional groups allowing a covalent connection between dye and silica network represents a method to minimize dye leaching [12, 13]. Well-known examples are fluorescein isothiocyanate (FITC) and rhodamine B isothiocyanate (RBITC), which can be functionalized in an addition reaction with a primary amine such as (3-aminopropyl)triethoxysilane (APTES) (Scheme 1) [14, 15].

During the synthesis the modified dyes can either be added to the reaction mixture $[16,17]$ or they can be attached to the surface of preformed silica particles $[14,15,18,19]$. In the latter case the dyes are exposed to environmental conditions and are not protected inside the particle, which can be avoided by formation of a protecting core-shell structure in a second step. For example, an outer silica shell around the fluorescent core can lead to an improved retention of dyes [10,20,21], an increase in the quantum yield, and an improved photostability compared to the free dye [20, 22-28]. Dyes without anchor groups can also be retained by using special precursors which increase the interaction between dye and silica network [10].

With regard to dye leaching and necessity of covalent dye incorporation, only a few detailed studies were published in the past [10-12, 29]. Since the particles are often used in sensitive biological matrices, it is necessary to obtain a sufficient understanding of the interaction between particle and dye. In addition to the leaching of the dyes, which is only investigated qualitatively in most studies, this understanding should also include the localization of the dyes in or on the particle and an investigation of the binding mode. Tolbert et al. showed that the extent of the dye leaching decreases as the strength of the dye's binding to the particle increases [11]. In our study, we studied leaching over a longer time period and investigated leaching-
Scheme 1 Rhodamine B and APTES functionalized rhodamine B isothiocyanate, disodium salt of fluorescein (uranine) and fluorescein isothiocyanate with APTES anchor group
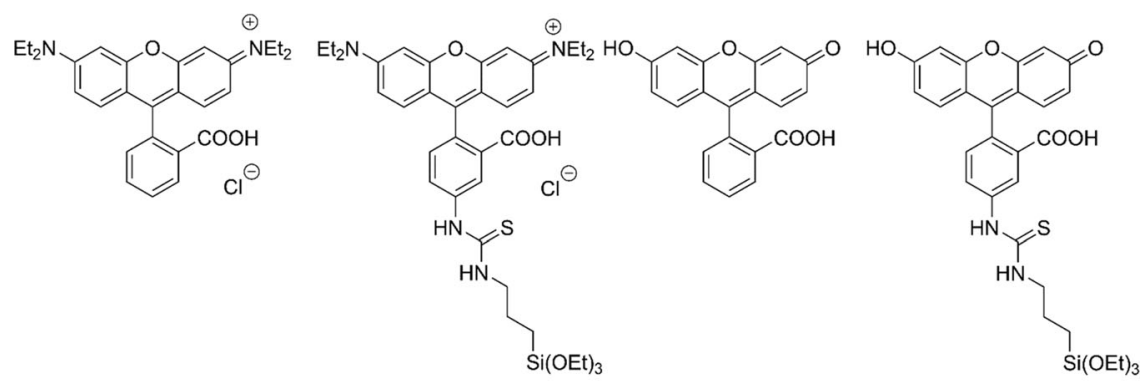

APTES functionalized RB
Uranine

APTES functionalized uranine 
influencing factors such as the intensity of the dye-particle interaction and the position of the dye molecules in the particle. Contrary to published results our focus was on the study of small silica nanoparticles with a diameter of less than or equal $40 \mathrm{~nm}$, as they are particularly interesting for various applications, for example in biology and medicine [30, 31]. As model systems we used rhodamine B and uranine due to the possibility of a straightforward chemical modification of the corresponding isothiocyanate derivates. Rhodamine B and uranine also differ in their charge. Rhodamine B as a single positively charged molecule can possibly interact more effectively than uranine with the negatively charged surface of the silica particles.

To protect the dye, it is common to condense a silica shell onto the core particles [20]. However, thin shells on sub-100 nm particles are inconvenient to be realized with the Stöber method [32]. Another possibility is the attachment of organosilane capping agents to the surface which we have chosen in the presented study.

\section{Experimental section}

Further characterization and methods can be found in the Supplementary Information (SI).

\subsection{Materials}

Fluorescein isothiocyanate isomer I (FITC) $\geq 90 \%$, rhodamine $\mathrm{B}$ isothiocyanate (RBITC) mixed isomers, rhodamine $\mathrm{B}(\mathrm{RB}) \geq 95 \%$, trimethoxy(octadecyl)silane (C18) $90 \%$ and chlorotrimethylsilane (TMS) $\geq 99 \%$ were purchased from Sigma-Aldrich (Steinheim, Germany). Uranine was obtained from Kremer Pigmente (Aichstetten, Germany), (3-aminopropyl) triethoxysilane (APTES) was obtained from Wacker Silicones (Burghausen, Germany), tetraethyl orthosilicate (TEOS) 99\%, 2-[methoxy(polyethyleneoxy)6-9propyl]trimethoxysilane (PEG) $90 \%$ and n-dodecyltrimethoxysilane (C12) $95 \%$ were provided by ABCR (Karlsruhe, Germany). Ammonium hydroxide solution $\approx 25 \%$ and all common solvents were purchased from the central chemical store of Saarland University. All chemicals were used as received without further purification.

\subsection{Methods}

Fluorescence emission and excitation spectra were measured with a FluoroMax-4 from HORIBA Jobin Yvon with a $150 \mathrm{~W}$ xenon arc-lamp at a $90^{\circ}$ angle in a type $10110 \times$ $10 \mathrm{~mm}$ quartz cuvette. Absorption spectra were measured with a dual beam PerkinElmer Lambda 750 UV/Vis/NIR Spectrometer; scattering samples were measured in transmittance mode in front of a $100 \mathrm{~mm}$ InGaAs Integrating Sphere. Fourier transform infrared (FTIR) spectra were recorded on a Bruker VERTEX 70 spectrometer. The samples were measured as dried powders at room temperature on a diamond-based attenuated total reflection (ATR) unit. The IR-spectra were recorded from 400 to $4500 \mathrm{~cm}^{-1}$ with a resolution of $4 \mathrm{~cm}^{-1}$ and averaged from 16 scans. Thermo gravimetric analysis (TGA) was performed using a Netzsch TG209 F1 Iris ASC in corundum crucibles. Adsorbed traces of solvent and water were removed by carrying out a previous heating step under nitrogen atmosphere from room temperature to $150^{\circ} \mathrm{C}$ with a heating rate of $10 \mathrm{~K} / \mathrm{min}$, holding the temperature for 10 minutes and subsequent cooling to room temperature. The actual temperature program starts by heating (10 $\mathrm{K} / \mathrm{min}$ ) from room temperature to $800{ }^{\circ} \mathrm{C}$ under nitrogen atmosphere followed by heating to $900{ }^{\circ} \mathrm{C}$ under nitrogen / oxygen atmosphere. Dynamic light scattering (DLS) was performed applying non-invasive backscattering using an ALV/CGS-3 compact goniometer system with an ALV/ LSE-5003 correlator at a $90^{\circ}$ goniometer angle. Samples from reaction solution were diluted, homogenized in an ultrasound bath, and equilibrated for about five minutes before measurement. For transmission electron microscopy (TEM) measurements, a JEOL JEM-2010 microscope was used. After dilution, $30 \mu \mathrm{L}$ of the sample was applied to a graphite coated copper grid (Plano S160-3) and measured after evaporation of the solvent. CP/MAS Solid State NMRs were performed at $25^{\circ} \mathrm{C}$ with a Bruker Ascend 400WB with a spinning rate of $13 \mathrm{kHz}$ and a delay time of $3-6 \mathrm{~s}$ (frequencies: ${ }^{13} \mathrm{C} 100.7 \mathrm{MHz}$ with adamantane as standard; ${ }^{29} \mathrm{Si} 79.5 \mathrm{MHz}$ with octakis(trimethylsiloxy)silsesquioxane as standard). CHN analysis was carried out on an Elementar Vario MICRO CUBE. Stationary fluorescence anisotropy measurements were performed on a Jasco FP-6500 at a $90^{\circ}$ angle with two angle adjustable polymer coated polarizer. Mean and standard deviation were determined in the range $\pm 20 \mathrm{~nm}$ around the anisotropy value at the emission maximum.

\subsection{Functionalization of xanthene dyes with 3- (Aminopropyl) triethoxysilane}

FITC and RBITC were functionalized with a triethoxysilane anchor group using literature procedures [14, 16]. $0.01 \mathrm{mmol}$ dye precursor (3.9 $\mathrm{mg}$ FITC or $5.4 \mathrm{mg}$ RBITC) were diluted in $4 \mathrm{~mL}$ ethanol and $0.50 \mathrm{mmol}(117 \mu \mathrm{L}, 0.111$ g) APTES were added. The reaction mixture was stirred overnight. The flask was kept in the dark to avoid photobleaching of the dye.

For use in the Stöber process, the obtained $2.5 \mathrm{mM}$ dye solution can be used directly. For later usage, the dye 
solutions must be stored in a freezer and must be used up within a few days.

\subsection{Preparation of fluorescent nanoparticles}

All fluorescent silica nanoparticles were prepared using a modified Stöber synthesis with dye concentrations of $0.4 \mathrm{mM}[6,16,33]$. As fluorescence anisotropy measurements could be affected by too high dye concentrations due to energy transfer phenomena such as Förster Resonance Energy Transfer (FRET) [34, 35], particles were also produced analogously to the procedure described below with a lower dye concentration $(0.002 \mathrm{mM})$.

\subsubsection{Dye concentration $0.4 \mathrm{mM}$}

In a $40 \mathrm{~mL}$ headspace vial, $22 \mathrm{~mL}$ ethanol, $3 \mathrm{~mL}$ ( $0.01 \mathrm{mmol})$ of the respective $3.3 \mathrm{mM}$ dye solution, $0.5 \mathrm{~mL}$ (2.26 mmol) TEOS and $0.75 \mathrm{~mL}(10.35 \mathrm{mmol}) 25 \%$ aqueous ammonia solution were mixed. After ten minutes, $0.5 \mathrm{~mL}(0.02 \mathrm{mmol})$ of an ethanolic $0.03 \mathrm{M} \mathrm{LiOH}$ solution was added dropwise under rapid stirring. The resulting dye concentration in the mixture thus is $0.4 \mathrm{mM}$. The mixtures were stirred at $275 \mathrm{rpm}$ for $24 \mathrm{~h}$. To the resulting opalescent dispersion, $27 \mathrm{~mL} n$-hexane was added for precipitation of the particles. The clear supernatant was carefully decanted, and the remaining particle dispersion was centrifugated at $8000 \mathrm{rpm}$ for ten minutes. The particles were washed three times with demineralized water and finally $80 \mathrm{mg}$ of particles were redispersed in $27 \mathrm{~mL}$ ethanol as stock solution (about $3 \mathrm{~g} / \mathrm{L}$ ) or were dried at $80^{\circ} \mathrm{C}$ in the vacuum for subsequent surface functionalization.

For the demobilization of dyes on the particle surface, first non-fluorescent Stöber particles are synthesized. After $24 \mathrm{~h}$ stirring, $4 \mathrm{~mL}$ of the respective $3.3 \mathrm{mM}$ dye solution was added to the Stöber mixture and stirred again for $24 \mathrm{~h}$. The workup was carried out analogously to the core particles.

For core-shell structure with dye only on the surface, first non-fluorescent Stöber particles are synthesized. After $24 \mathrm{~h}$ stirring, $4 \mathrm{~mL}$ of the respective $3.3 \mathrm{mM}$ dye solution was added to the Stöber mixture and stirred again for $24 \mathrm{~h}$. The workup was carried out analogously to the core particles.

Yield: $\sim 80 \mathrm{mg}$ particles, DLS: $\mathrm{SiO}_{2} 15.4 \pm 0.3 \mathrm{~nm}$, uranine-modified silica $25.9 \pm 1.2 \mathrm{~nm}$, FITC-modified silica $24.4 \pm 1.3 \mathrm{~nm}$, RB-modified silica $19.3 \pm 1.3 \mathrm{~nm}$, RBITCmodified silica $27.5 \pm 1.0 \mathrm{~nm}$, FT-IR: 3600-3000, 1050, 940, $792 \mathrm{~cm}^{-1}$, TGA: $95 \%$ residual mass, $\lambda_{\text {exc }}$ : uraninemodified silica $497 \mathrm{~nm}$, FITC-modified silica $502 \mathrm{~nm}$, RBmodified silica $541 \mathrm{~nm}$, RBITC-modified silica $549 \mathrm{~nm}$, $\lambda_{\text {em }}$ : uranine-modified silica $514 \mathrm{~nm}$, FITC-modified silica $522 \mathrm{~nm}$, RB-modified silica $565 \mathrm{~nm}$, RBITC-modified silica $568 \mathrm{~nm}$, fluorescence anisotropy $\mathrm{r}$ : uranine-modified silica 0.04, FITC-modified silica 0.14, RB-modified silica 0.04,
RBITC-modified silica 0.13 (solvent in all cases: ethanol), ${ }^{13} \mathrm{C}$-CP/MAS: $\delta=16.68,58.57 \mathrm{ppm},{ }^{29} \mathrm{Si}-\mathrm{CP} / \mathrm{MAS}: \delta=$ $-110.14,-101.87,-92.53 \mathrm{ppm}$.

\subsubsection{Dye concentration $0.002 \mathrm{mM}$ (for fluorescence anisotropy measurements)}

In a $40 \mathrm{~mL}$ headspace vial, $25 \mathrm{~mL}$ ethanol, $16.4 \mu \mathrm{L}\left(5.4 * 10^{-5}\right.$ mmol) of the respective xanthene $3.3 \mathrm{mM}$ dye solution, $0.5 \mathrm{~mL}(2.26 \mathrm{mmol})$ TEOS and $0.75 \mathrm{~mL}(10.35 \mathrm{mmol}) 25 \%$ ammonia solution were mixed. After ten minutes, $0.5 \mathrm{~mL}$ ( $0.02 \mathrm{mmol}$ ) of an ethanolic $0.03 \mathrm{M} \mathrm{LiOH}$ solution was added dropwise under rapid stirring. The resulting dye concentration in the mixture thus is $0.002 \mathrm{mM}$. The further procedure is analogous to Section 2.4.1.

Yield: about $80 \mathrm{mg}$ of particles, FT-IR: $3600-3000$, $1050,940,792 \mathrm{~cm}^{-1}, \lambda_{\text {exc }}$ : uranine-modified silica $497 \mathrm{~nm}$, FITC-modified silica $502 \mathrm{~nm}$, RB-modified silica $541 \mathrm{~nm}$, RBITC-modified silica $549 \mathrm{~nm}, \lambda_{\mathrm{em}}$ : Uranine-modified silica $514 \mathrm{~nm}$, FITC-modified silica $522 \mathrm{~nm}$, RB-modified silica $565 \mathrm{~nm}$, RBITC-modified silica $568 \mathrm{~nm}$, fluorescence anisotropy $\mathrm{r}$ : uranine-modified silica $0.028 \pm 0.002$, FITCmodified silica $0.227 \pm 0.004$, RB-modified silica $0.037 \pm$ 0.002 , RBITC-modified silica $0.352 \pm 0.009$ (solvent for all measurements: ethanol).

\subsubsection{Esterification of rhodamine B on the silica surface}

Following a modified literature method [36], $1 \mathrm{~g}$ unmodified silica particles and $100 \mathrm{mg}$ RB were dissolved in $100 \mathrm{~mL}$ isobutylmethyl ketone (IBMK) and heated at $100^{\circ} \mathrm{C}$ overnight. After cooling to room temperature, the particles were separated from IBMK via centrifugation at $8000 \mathrm{rpm}$ and washed with ethanol six times in $10 \mathrm{kDa}$ membrane concentrators. The still strongly colored particles were dried at $80^{\circ} \mathrm{C}$ under vacuum.

\subsubsection{Preparation of alkylated core-shell particles}

The functionalization was carried out following a modified literature method [37]. To obtain a protecting organic layer on the particle surface, four common functionalization agents with different chain lengths were tested. In a headspace vial, $100 \mathrm{mg}$ of silica particles with esterified rhodamine B were dried for ten minutes in high vacuum, dispersed in $10 \mathrm{~mL}$ absolute toluene, and then stirred for ten minutes. $0.04 \mathrm{mmol}$ (5.1 $\mu \mathrm{L}$ trimethyl silyl chloride (TMSCl), $13.1 \mu \mathrm{L}$ dodecyltrimethoxysilane $(\mathrm{C} 12), \quad 15.8 \mu \mathrm{M}$ octadecyltrichlorosilane (C18), $10.8 \mu \mathrm{L}$ 2-[methoxy(polyethyleneoxy)6-9propyl]trimethoxysilane (PEG)) of the respective functionalization agent were added dropwise under quick stirring. The reaction mixtures were heated to $70{ }^{\circ} \mathrm{C}$ for $24 \mathrm{~h}$. After cooling down, the dispersions were centrifugated at $8000 \mathrm{rpm}$ and washed 


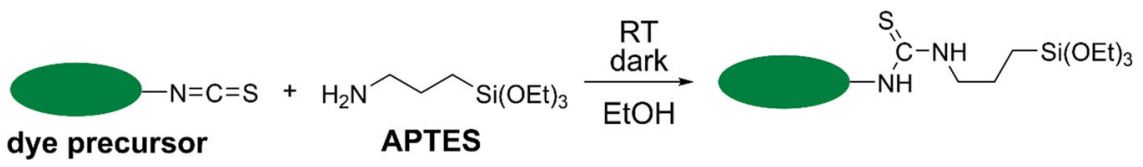

Scheme 2 Functionalization of xanthene precursors RBITC and FITC with the triethoxysilane APTES for later use in Stöber process

three times with fresh toluene. Then, the particles were dried at $80{ }^{\circ} \mathrm{C}$ in the vacuum for $24 \mathrm{~h}$.

Yield: TMS-modified silica $98 \mathrm{mg}, \mathrm{C} 12$-modified silica $92 \mathrm{mg}$, C18-modified silica $85 \mathrm{mg}$ particles, PEG-modified silica $91 \mathrm{mg}$, DLS: TMS-modified silica $26.7 \pm 2.4 \mathrm{~nm}$, C12-modified silica $29.8 \pm 2.7 \mathrm{~nm}$, C18-modified silica $39.4 \pm 5.4$ nm, FT-IR: 3600-3000, 2980-2850, 1395-1460, 1050, 940, $792 \mathrm{~cm}^{-1}$, TGA: TMS-modified silica $92.1 \%$, C12-modified silica $89.1 \%$, C18-modified silica $85.8 \%$, ${ }^{13} \mathrm{C}-\mathrm{CP} / \mathrm{MAS}$ : TMS-modified silica $\delta=-1.51 \mathrm{ppm}, \mathrm{C} 12 /$ C18-modified silica $\delta=11.70,21.85,29.05,31.49$, $42.06 \mathrm{ppm},{ }^{29} \mathrm{Si}-\mathrm{CP} / \mathrm{MAS}$ : TMS-modified silica $\delta=$ $-110.95, \quad-101.87, \quad-93.47$. 12,92 (M $\left.\mathrm{M}_{1}\right)$ ppm, C12modified silica $\delta=-110.99,-101.87,-93.56,-67.47$ $\left(\mathrm{T}_{3}\right),-57.39\left(\mathrm{~T}_{2}\right)$ ppm, C18-modified silica $\delta=-110.43$, $-101.87,-92.84,-66.69,-57.25$ ppm, PEG-modified silica $\delta=-110.74,-101.87,-93.26,-58.92 \mathrm{ppm}$.

\subsection{Leaching tests}

For leaching analysis, $2 \mathrm{~mL}$ of the particle stock solution were diluted with $17 \mathrm{~mL}$ ethanol and stirred with $275 \mathrm{rpm}$ at room temperature in sealed headspace vials. Every day, $3 \mathrm{~mL}$ of the particle suspension were removed via syringe through the septum to avoid changes in concentration which could be due to evaporation of the solvent. The samples were then centrifugated at $8000 \mathrm{rpm}$ for $10 \mathrm{~min}$ and filtered with $0.2 \mu \mathrm{m}$ syringe filters to remove the bulk (about $75 \%$ ) of the particles. Complete separation of the particles can be achieved with $10 \mathrm{kDa}$ membrane concentrators (Corning ${ }^{\circledR}$ Spin- $\mathrm{X}^{\circledR}$ UF concentrators), which were only used in the last step of the leaching process to verify leaching results. The absorbance of the centrifugate was measured and compared with the absorbance intensity of the particle dispersion. All samples were measured in front of an integrating sphere to minimize scattering effects of particles. Remaining scattering effects were removed from the spectra by a baseline correction that takes Rayleigh scattering into account (detailed information in the supporting info) [38]. To ensure reproducibility, all leaching experiments were carried out as triplicates in disposable cuvettes.

To check the effect of protecting shells, the oven dried functionalized particles were dispersed in ethanol $(3 \mathrm{mg} / \mathrm{mL})$ and stirred for $24 \mathrm{~h}$ at $275 \mathrm{rpm}$. The rest of the procedure was the same as for unfunctionalized particles.

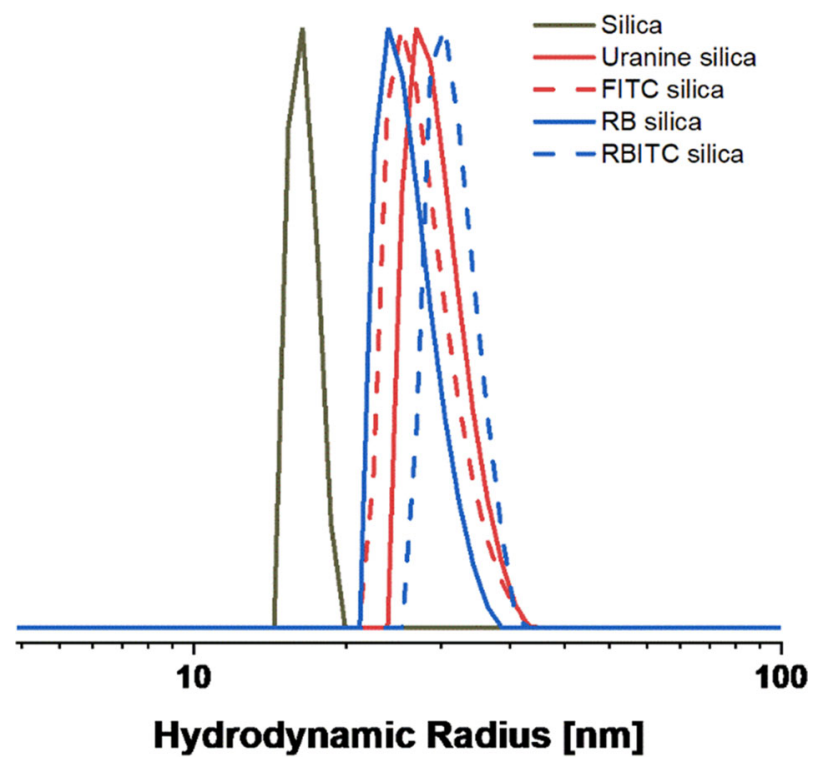

Fig. 1 DLS characterization of the fluorescent particles and pure silica

\section{Results and discussion}

\subsection{Dye precursors and particle characterization}

\subsubsection{Dye precursors}

To compare the effect of physical entrapment in the particle core compared to covalent bonding, the xanthene dyes fluorescein and rhodamine B and their isothiocyanate derivates were chosen because of the option for a straightforward modification as well their good solubility in polar solvents like alcohols, which is important for usability in the Stöber-process. In this work, the isothiocyanate precursors FITC and RBITC were modified with (3-aminopropyl) triethoxysilane (APTES) using a modified literature procedure (Scheme 2) [14, 16].

\subsubsection{Particle size and shape}

The nature of dye binding seems to play only a small role for particle sizes. Particles with any kind of dye label tend to be slightly larger than pure silica particles (Fig. 1), which is consistent with literature results [11].

The pure silica particles show a diameter of $24.1 \pm$ $2.9 \mathrm{~nm}$. The particles with physisorbed dyes are larger in size with $39.2 \pm 4.6 \mathrm{~nm}$ for uranine silica and $37.8 \pm 3.8 \mathrm{~nm}$ 

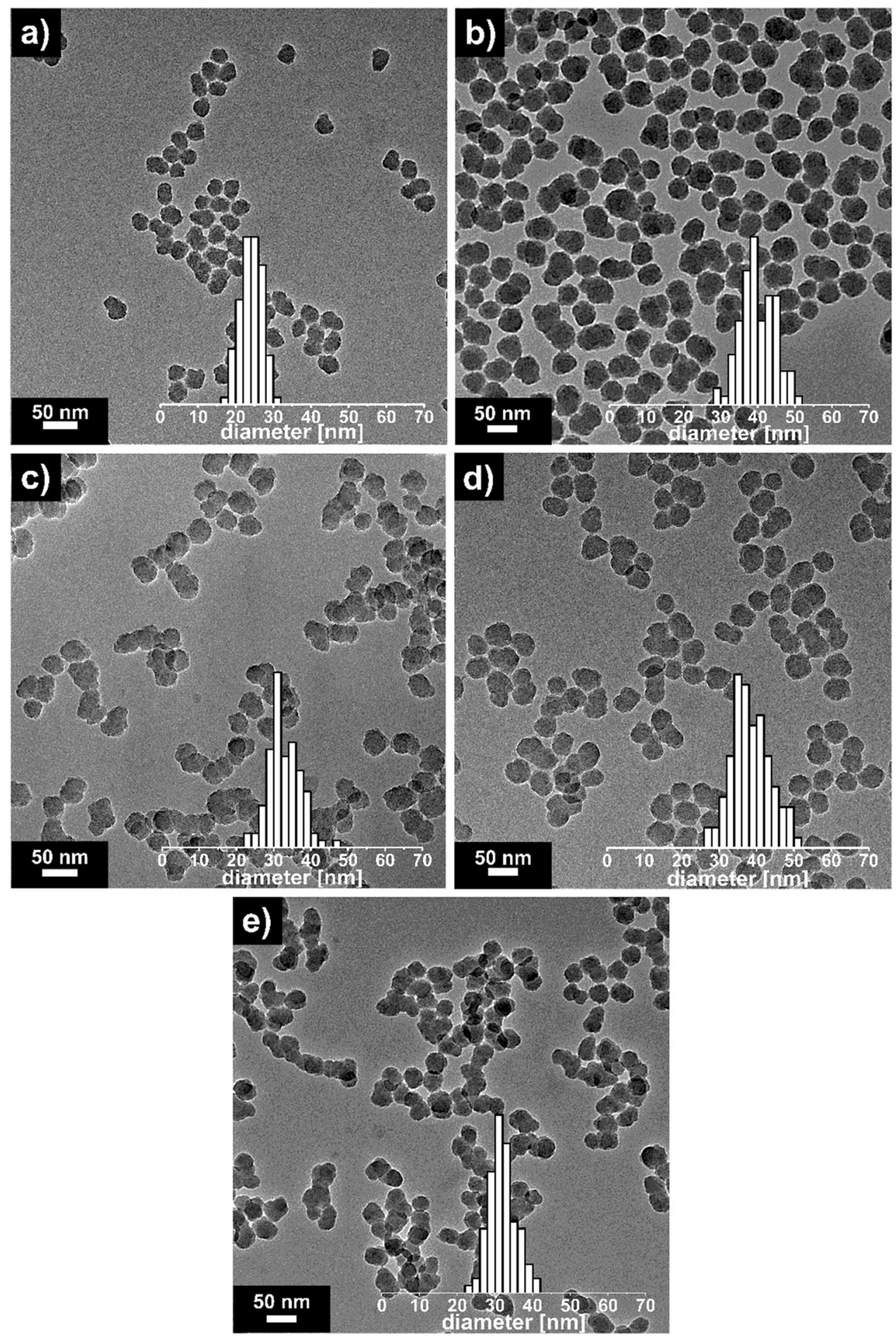

Fig. 2 TEM pictures with size distribution of all synthesized silica core particles. The size distribution was determined by measuring the diameter of 100 particles. a silica, b uranine-modified silica, c FITC-modified silica, d RB-modified silica, e RBITC-modified silica

for rhodamine B silica, which was not apparent from the DLS measurements. With $33.0 \pm 4.2 \mathrm{~nm}$ and $31.9 \pm 3.5 \mathrm{~nm}$, FITC-modified silica and RBITC-modified silica are smaller than the particles with non-covalently attached dyes, but larger than completely unfunctionalized particles.
The increase in diameter for silica particles with covalently bonded FITC or RBITC can be explained by decrease of the particle density and by destabilization of the initially formed oligomers at the beginning of the sol-gel reaction. This destabilization is caused by the compensation of the charge 
of $\mathrm{Si}-\mathrm{O}^{-}$by free ammonium groups (at $\mathrm{pH}$ values $>2$ ) from the excess APTES used for dye functionalization [39].

The TEM images (Fig. 2) show particles with uniform morphology. The polydispersity ranges from 11.0 to $13.5 \%$ and are in the normal range for small Stöber particles [15, 40].

After synthesis, the particles were washed three times with distilled water to remove non-reacted precursors and non-bonded/incorporated dyes. During these washing steps it could already be observed by eye that the dyes without covalent attachment (uranine and rhodamine B) showed a strong dye loss.

Due to the influence of the solvation shell, diameters determined with TEM are slightly smaller than those measured with DLS (Table 1), which is in agreement with literature results [41-43].

\subsubsection{Spectroscopic properties of the particles}

All dye doped particles emitted in the green (fluorescein dyes) to yellow (rhodamine B dyes) part of the visible light spectrum (Fig. 3). Uranine and FITC are excited at $500 \mathrm{~nm}$ and emit at $522 \mathrm{~nm}$. A PBS ( $\mathrm{pH}=7.4$ ) buffer was used due to $\mathrm{pH}$ dependency of fluorescein dyes.

The corresponding nanoparticles behave similarly and show only small deviations in shift or shape of the

Table 1 Comparison between hydrodynamic diameters and particle diameters obtained from TEM

\begin{tabular}{lll}
\hline Nanoparticle sample & DLS: $\mathrm{d}_{\mathrm{H}} \pm \sigma[\mathrm{nm}]$ & TEM: $\mathrm{d} \pm \sigma[\mathrm{nm}]$ \\
\hline Silica & $31 \pm 1$ & $24.1 \pm 2.9$ \\
Uranine-modified silica & $52 \pm 1$ & $39.2 \pm 4.6$ \\
FITC-modified silica & $49 \pm 3$ & $33.0 \pm 4.2$ \\
Rhodamine B-modified silica & $46 \pm 1$ & $37.8 \pm 3.8$ \\
RBITC-modified silica & $55 \pm 2$ & $31.9 \pm 3.5$ \\
\hline
\end{tabular}

spectrum. Rhodamine B labeled particles have the same maxima as the free dye (excitation at $541 \mathrm{~nm}$, emission at $565 \mathrm{~nm}$ ). RBITC shows the same maxima positions as RB, but with a small red shift of the associated nanoparticle spectra $\left(\lambda_{\text {exc }}=549 \mathrm{~nm}, \lambda_{\mathrm{em}}=568 \mathrm{~nm}\right)$. This red shift may be due to $\pi$-stacking effects of local concentrated dye molecules which can result in reabsorption or excimer formation [39].

After washing, the particles were dispersed in $50 \mathrm{~mL}$ of fresh ethanol or dried for subsequent functionalization. The dispersions are stable for several weeks and can be used as stock solutions.

\subsection{Validation of binding character and dye distribution in the particle}

For leaching studies, the actual position of the attached dye is important, because dyes near the particle surface are more exposed to the solvent and other small molecules than dyes inside of the particle. Montalti et al. showed that the thickness of the solvent exposed shell is independent of the particle size and was determined to be around $3-4 \mathrm{~nm}$ for Stöber particles [44]. In order to determine whether the xanthene dyes are bound to the surface or are more likely embedded inside the particle, core-shell particles with the dye only on the surface were prepared in addition to the standard Stöber particles (Scheme 3).

The surrounding matrix influences the spectroscopic properties of rhodamine $\mathrm{B}$ and uranine. Uranine exists in several forms depending on the $\mathrm{pH}$ value [45]. The dianionic species shows the strongest absorption, while the neutral lactone form is, if at all, only slightly colored [46]. Through the other substitution pattern, rhodamine B only exists in three possible species: as cation or one of two neutral species (a zwitterion or a uncolored lactone) [45].
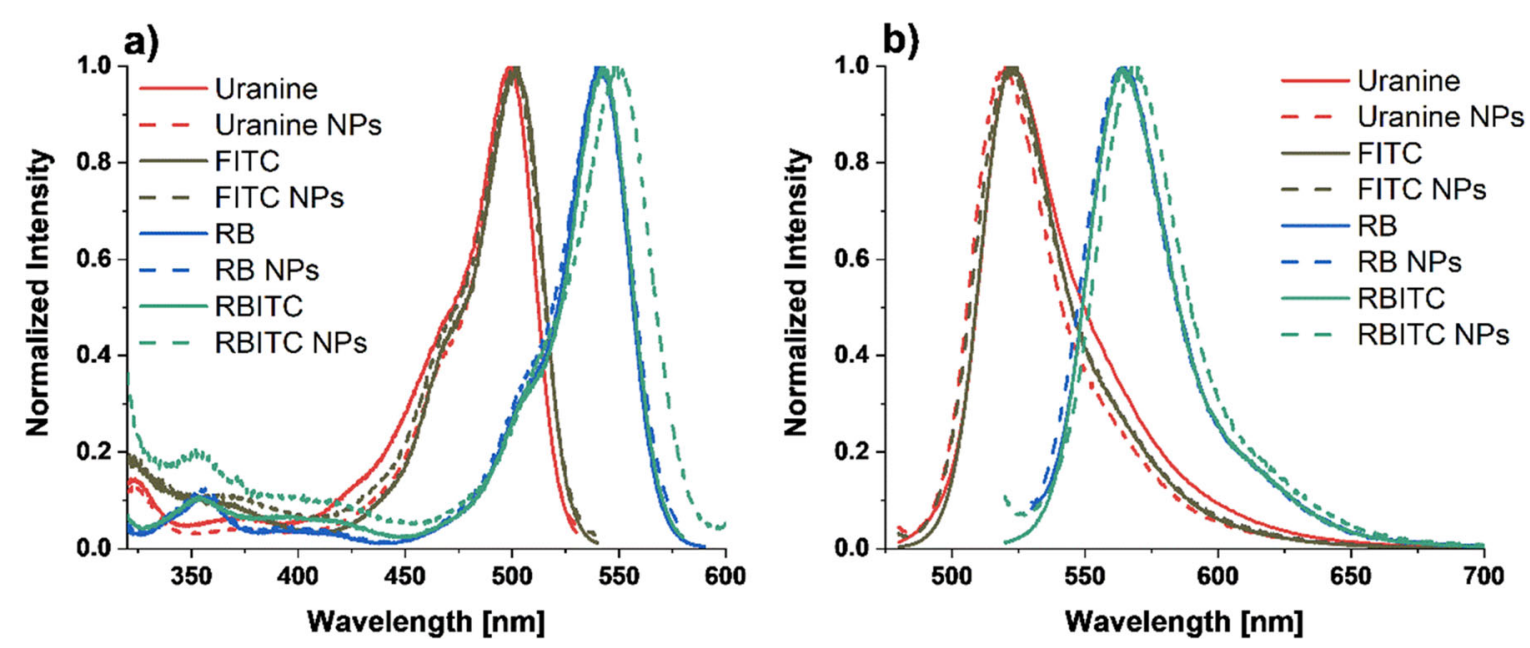

Fig. 3 a Excitation and b Emission spectra of rhodamine B and fluoresceine doped particles and the free dyes in PBS buffered solution 
Scheme 3 Different particle morphologies for dye localization. a Standard Stöber particle with direct dye addition, b Core-shell structure by subsequent addition of the dye

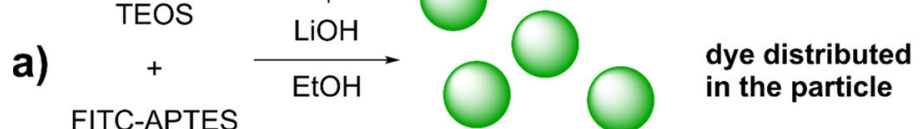

b)

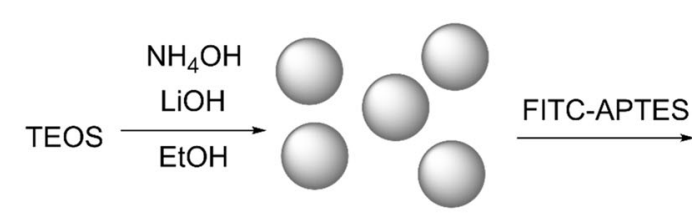

bare silica

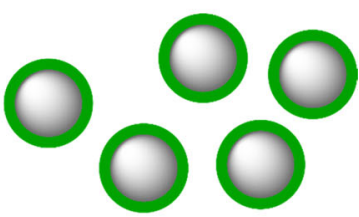

dye on the surface

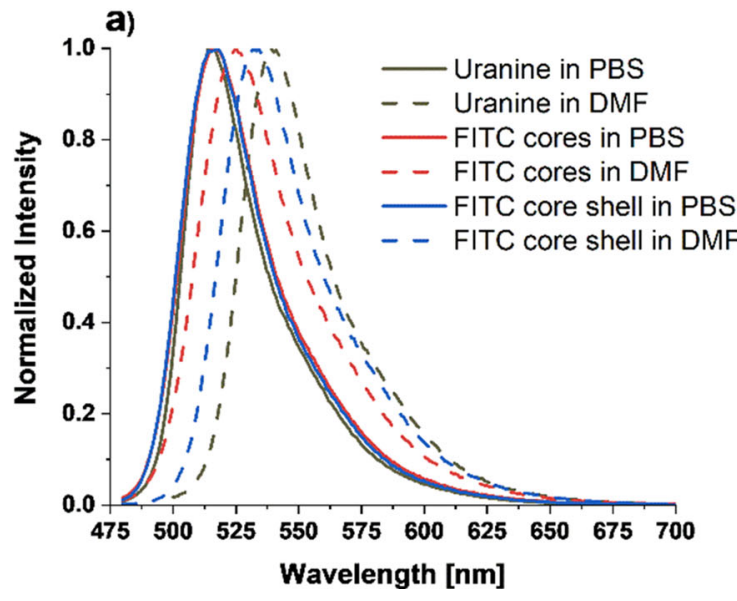

Fig. 4 Spectroscopic characterization of free rhodamine $B(R B)$ and uranine, the core- and core-shell particles in PBS buffer $(\mathrm{pH}=7.4)$ and DMF. a Fluorescence emission spectra of free uranine and

Fluorescence excitation and emission spectra of the free dyes, the core particles, and the core-shell particles were measured in a phosphate-buffered saline (PBS buffer) at $\mathrm{pH}$ 7.4 and in DMF (Fig. 4). The change of the solvent should effect the spectra of the dyes on the surface, while particles with dyes in the interior should show no or only a minor solvent influence [47].

In the case of uranine, the free dye shows a clear red shift of the emission from $515 \mathrm{~nm}$ in PBS to $539 \mathrm{~nm}$ in DMF (Fig. 4a). The core-shell particles behave like the free uranine and show a red shift from $518 \mathrm{~nm}$ in PBS to $532 \mathrm{~nm}$ in DMF due to their solvent exposed dye molecules. Van Blaaderen et al. determined for particles with exposed FITC shell in DMF a similar wavelength [14]. The emission of FITC core particles only shifts from 516 to $525 \mathrm{~nm}$. In the case of FITC doped core particles, this experiment leads to the assumption that FITC molecules are mainly distributed in the inner particle. Due to the slight red shift, a small part of the dye molecules seems to be in the solvent exposed shell.

The particles with rhodamine B show an opposite solvatochromic behavior. Free rhodamine B in phosphatebuffered saline (PBS buffer) emits at $576 \mathrm{~nm}$, and in DMF /

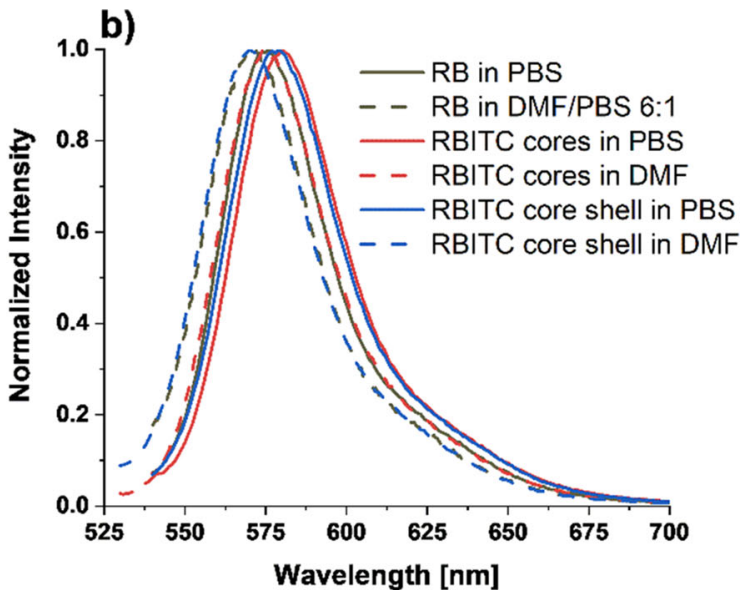

particles $\left(\lambda_{\text {exc }}=470 \mathrm{~nm}\right), \mathbf{b}$ Emission spectra of free rhodamine B and particles $\left(\lambda_{\text {exc }}=520 \mathrm{~nm}\right)$

PBS (6:1) at $571 \mathrm{~nm}$ (Fig. 4b). Rhodamine B in DMF had to be mixed with PBS because the lactone formed in pure DMF shows no measurable fluorescence. The blue shifts of free rhodamine B fluorescence emission spectra in PBS with increasing DMF content are shown in Fig. 5.

The blue-shift is strongest for the core-shell particles from 579 to $570 \mathrm{~nm}$; the regular Stöber particles show only a shift from 580 to $574 \mathrm{~nm}$. Overall, the difference in the solvent dependent shift for the emission spectra of rhodamine B is smaller than that of uranine, but it can be observed that the core-shell particles show the greatest similarity to the free dye. In summary, FITC and RBITC seem to be distributed both inside and partially outside the core particles.

In addition to the position of the dye in the particle, the covalent or non-covalent nature of the dye-particle connection should be proven, as well as the strength of the dye-particle interaction. Due to low dye concentrations, statements about the strength of the dye binding are only possible with spectroscopic methods such as fluorescence anisotropy [47]. In this method, fluorophores get excited by polarized light. The fluorophores with a transition moment that is parallel to the electric vector of the excitation light absorb the light, get 

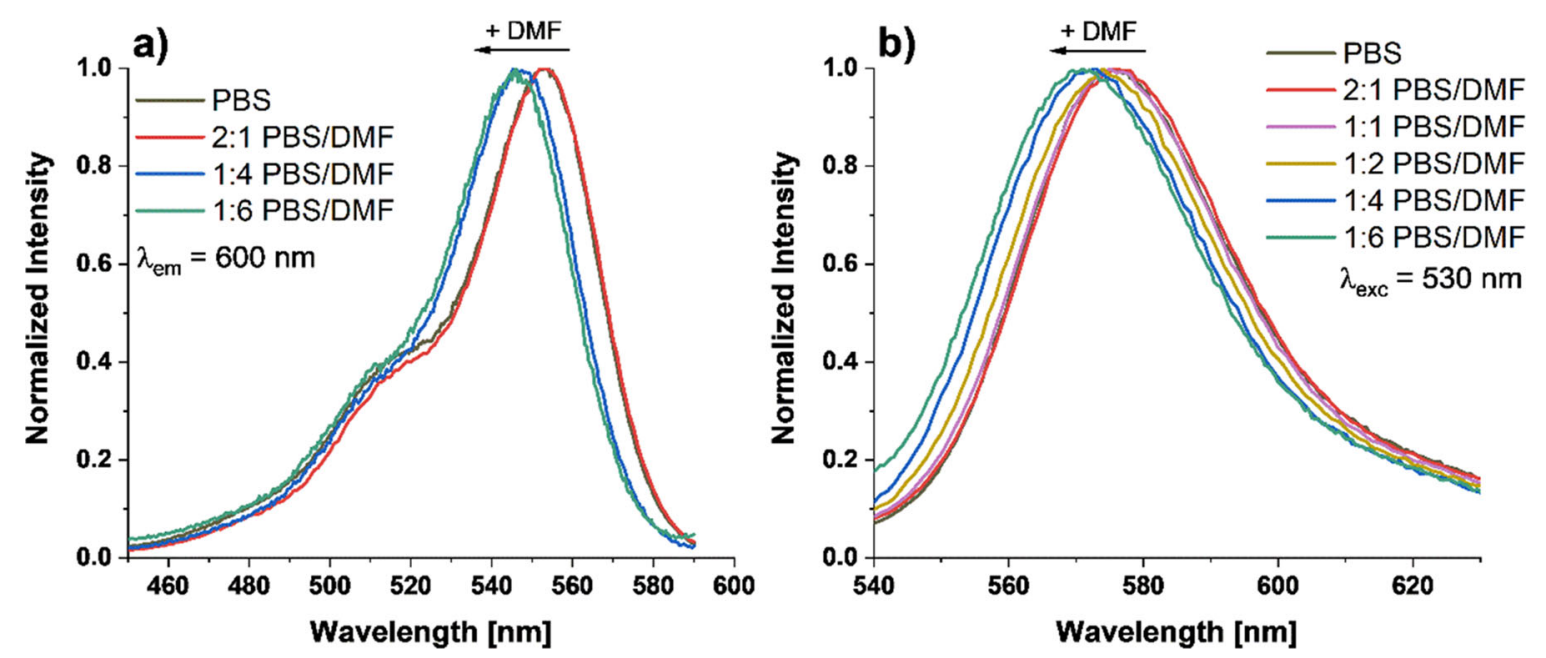

Fig. 5 Blue shift of the fluorescence $\mathbf{a}$ excitation and $\mathbf{b}$ emission spectra of rhodamine B in PBS buffer with increasing addition of DMF
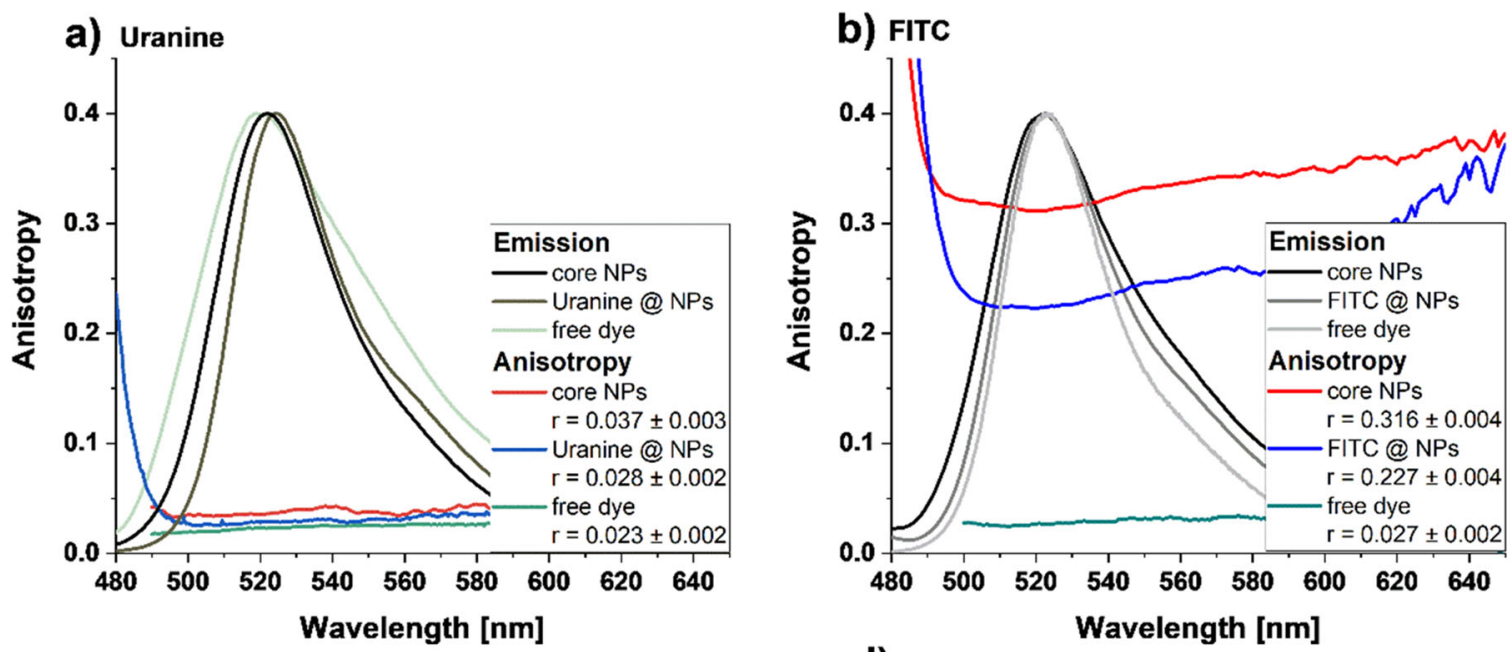

C) Rhodamine B
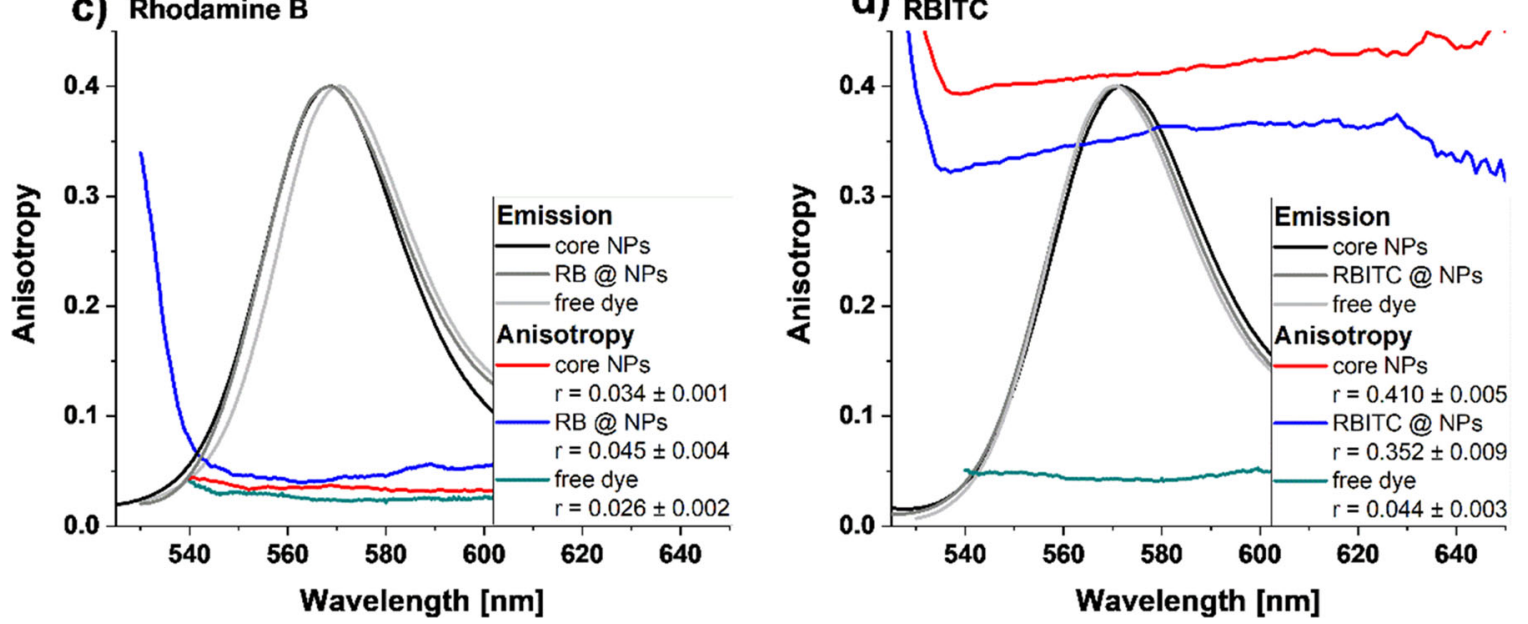

Fig. 6 Fluorescence anisotropy measurements of different xanthene particles and free dyes in ethanol. The anisotropy values of covalently attached dyes are clearly higher than those of the physically attached dyes

excited and emit polarized light. Depolarization of isolated fluorophores occurs when rotational diffusion during the fluorescence lifetime takes place. As a result, dyes that are in rigid structures (e.g., through covalent connection or strong physical interactions with the environment) have a higher anisotropy value than more mobile dyes [48]. Theoretically, 
the anisotropy value of a randomly oriented sample can range from -0.20 to 0.40 .Maximum values of about 0.37 have been found experimentally for dyes of the xanthene family with low mobility [49], but also values over 0.40 are possible if scattering effects are involved [50]. The fluorescence anisotropy is defined as $r$ (Eq. 1) [50], where $I_{\|}$and $I_{\perp}$ are the vertically and horizontally polarized emissions excited by vertically polarized light.

$r=\frac{\mathrm{I}_{\|}-\mathrm{I}_{\perp}}{\mathrm{I}_{\|}+2 \mathrm{I}_{\perp}}$

The measurements clearly show a difference between the dyes with or without the possibility to connect covalently to the silica network and between the different particle morphologies (Fig. 6).

Anisotropy values for the core particles including dyes with anchor group are significantly higher than for the core particles with physisorbed dyes, whose anisotropy values correspond approximately to those of the free dyes. The value for the RBITC-cores is slightly above the theoretical maximum of the anisotropy of 0.4 . This can probably be explained by the fact that with particulate samples there is always reflection and scattering of the light, which influences the intensity of the measured fluorescence [50]. An overview of all fluorescence anisotropic measurements is given in Table S1.

The interaction of the physisorbed dyes is therefore very weak, while the high values of the anchor groupcontaining dyes suggest a strong, i.e., covalent, interaction.
The anisotropies of FITC and RBITC on the particle surface (core-shell NPs) are lower than those of the core nanoparticles, which can be explained by the better mobility of the dye molecules outside the silica network [51]. The comparison of the anisotropy values are therefore another indication of a homogeneous distribution of the dyes in the core nanoparticles. The anisotropies of the core-shell NPs with physisorbed dyes again correspond approximately to those of the free dyes and thus also appear to be exposed only to very weak interactions with the silica surface.

\subsection{Organic protection layer}

To reduce leaching of physisorbed dyes, organic functionalizations for the particle surface were carried out. Four different capping agents were used to tailor the surface in different ways. As lipophilic functionalizations, we applied trimethylsilyl chloride (TMSCl), dodecyl(trimethoxy)silane $\left(\mathrm{C}_{12}\right)$, octadecyl(trimethoxy)silane $\left(\mathrm{C}_{18}\right)$ and as hydrophilic functionalization 2-[methoxy (polyethyleneoxy)6-9propyl]trimethoxysilane (PEG). Direct surface modification of particles with adsorbed dye leads to a strongly decreased fluorescence intensity of the particles due to replacement of the surface-adsorbed dye molecules by the functionalization agent. To preserve the emission performance, the dye molecules can be anchored on the surface via esterification between the carboxylic acid group of the dye and the silanol groups on the particle surface (Scheme 4) [52].
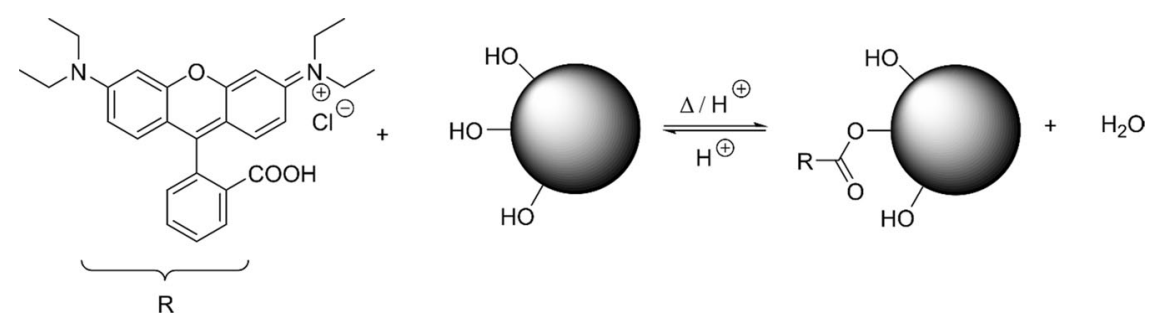

Scheme 4 Esterification equilibrium of the dye bearing the carboxylic acid group and the silica particles. The equilibrium can be shifted to the right side by addition of acid/heat or by the presence of a educt or product in excess or deficit
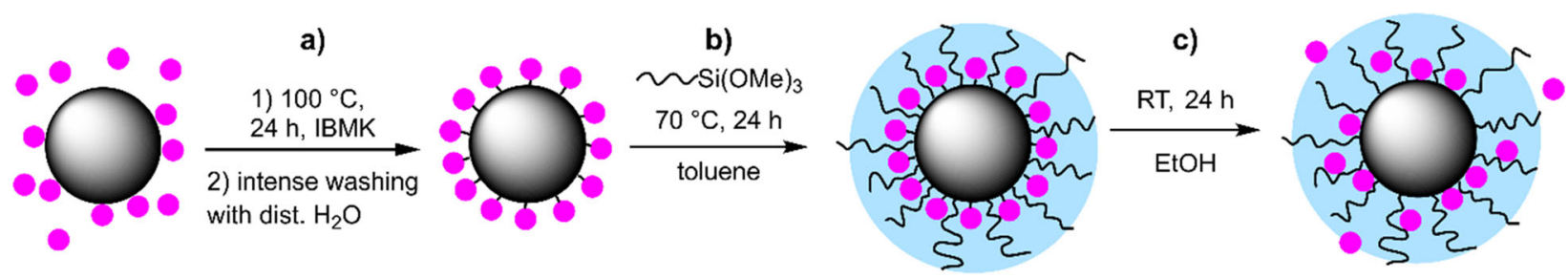

Particle and

Rhodamine B

Scheme 5 Modification of rhodamine B-modified silica NPs without strong dye loss. a particles are heated with RB to induce esterification, $\mathbf{b}$ addition of a protecting shell $\mathbf{c}$ redispersion in solvents containing water induces ester cleavage 
The esterification is carried out before the addition of the functionalization agent and is conducted at high temperatures (Scheme 5a).

After thorough washing of the particles, they can be functionalized with the respective surface functionalizing reagent (Scheme 5b). Since an esterification is an equilibrium reaction, the ester bond is split again, and the dyes are retained only by the applied shell in the subsequent leaching experiments (Scheme 5c).

The inset in Fig. 7 shows the successful dye doping after intense particle washing because there are still visible dye vibrations such as the ester / carboxylic acid $\mathrm{C}=\mathrm{O}$ vibration at $1691 \mathrm{~cm}^{-1}$.

\subsection{Characterization of the core-shell particles}

Since the xanthene molecules are polar, addition of a lipophilic particle shell should be advantageous to avoid leaching phenomena. In addition, the steric demand of this surface functionalization could further improve dye retention. For the functionalization the dry particles were dispersed in absolute toluene, heated up to $70^{\circ} \mathrm{C}$ and the respective functionalizing agent was added dropwise under an inert gas atmosphere [5]. Upon subsequent heating, a better dispersibility of the particles in the apolar solvent can already be observed. After 24 $\mathrm{h}$, the particles were separated from the solvent through centrifugation and washed with toluene.

Successful functionalization of the particles can be detected by DLS, FT-IR, TGA and CP/MAS NMR spectroscopy. Dynamic light scattering measurements of the dispersed particles show an increase of the hydrodynamic radii depending on the alkyl functionalization. For functionalization, the particles in ethanol must be dried and then redispersed in toluene. For comparison with the previous core particles, the dried particles were redispersed in ethanol

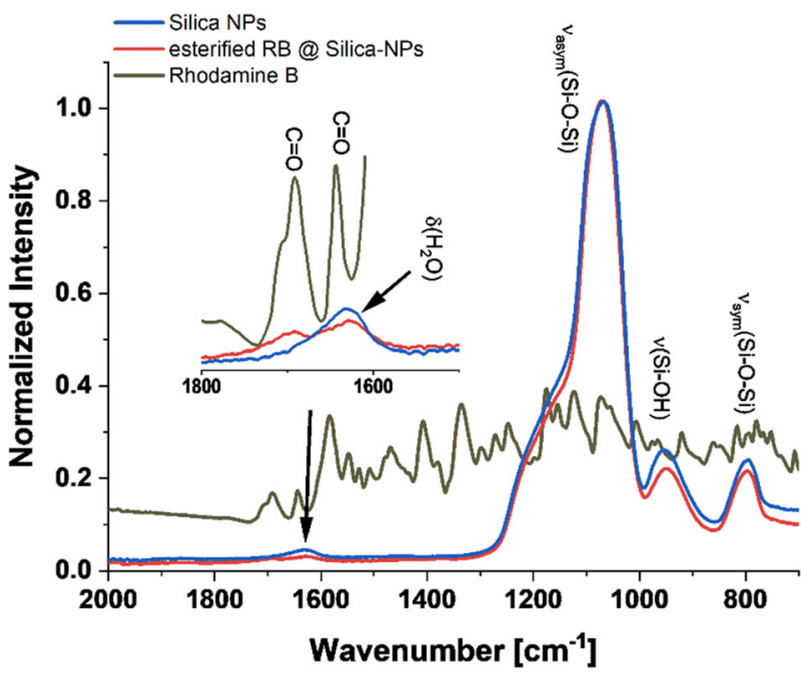

Fig. 7 FT-IR spectra of the esterified rhodamine B particles for DLS measurement. Drying and redispersion of silica particles result in a slight increase of the hydrodynamic radius from $19.3 \pm 1.4 \mathrm{~nm}$ to $24.3 \pm 1.9 \mathrm{~nm}$ (Fig. 8). Functionalization with TMS results in only slightly larger radii $(26.7 \pm 2.4 \mathrm{~nm})$, while the longer alkyl chains increase the radius more strongly $(\mathrm{C} 12: 29.8 \pm 2.7 \mathrm{~nm}, \mathrm{C} 18: 39.4 \pm$ $5.4 \mathrm{~nm}$, PEG: $41.8 \pm 6.9 \mathrm{~nm}$ ) and, in the case of the C18 and PEG groups, also lead to a broadening of the size distribution.

In the FT-IR spectra of all silica particles, between $3000 \mathrm{~cm}^{-1}$ and $3600 \mathrm{~cm}^{-1} \mathrm{O}-\mathrm{H}$ vibrations of adsorbed water and $\mathrm{OH}$ surface groups can be observed (Fig. 9). Characteristic for silica is the antisymmetric $\mathrm{Si}-\mathrm{O}-\mathrm{Si}$ vibration at $1050 \mathrm{~cm}^{-1}, \mathrm{Si}-\mathrm{OH}$ vibration at $940 \mathrm{~cm}^{-1}$ and the symmetric Si-O-Si vibration at $792 \mathrm{~cm}^{-1}$ [14]. The alkyl functionalization can be shown by the $\mathrm{C}-\mathrm{H}$ - oscillations between $2850 \mathrm{~cm}^{-1}$ and $2980 \mathrm{~cm}^{-1}$ and the C-H deformation oscillations between $1395 \mathrm{~cm}^{-1}$ and $1460 \mathrm{~cm}^{-1}$ [15]. The small signal at $848 \mathrm{~cm}^{-1}$ can be assigned to $\mathrm{Si}-\mathrm{CH}_{3}$ vibrations of the successful TMS functionalization; [16] the corresponding $\mathrm{C}-\mathrm{H}$ vibrations are not visible due to the low molecular weight of TMS.

In TGA measurements, the varying organic content of the particles can be shown (Fig. 10 and Table 2).

Pure silica particles have only a very small mass loss of about $5 \%$ when heated to $900{ }^{\circ} \mathrm{C}$, which can be caused by traces of trapped solvent, adsorbed water and condensation of surface groups (Si-OH and Si-OEt) [17, 18]. The dyes are also not visible in TGA due to their low concentration. The functionalized particles begin to lose mass at around $200{ }^{\circ} \mathrm{C}$ due to the decomposition of the organic groups. The residual mass of pure RB-silica is $95.3 \%$; the residual masses of

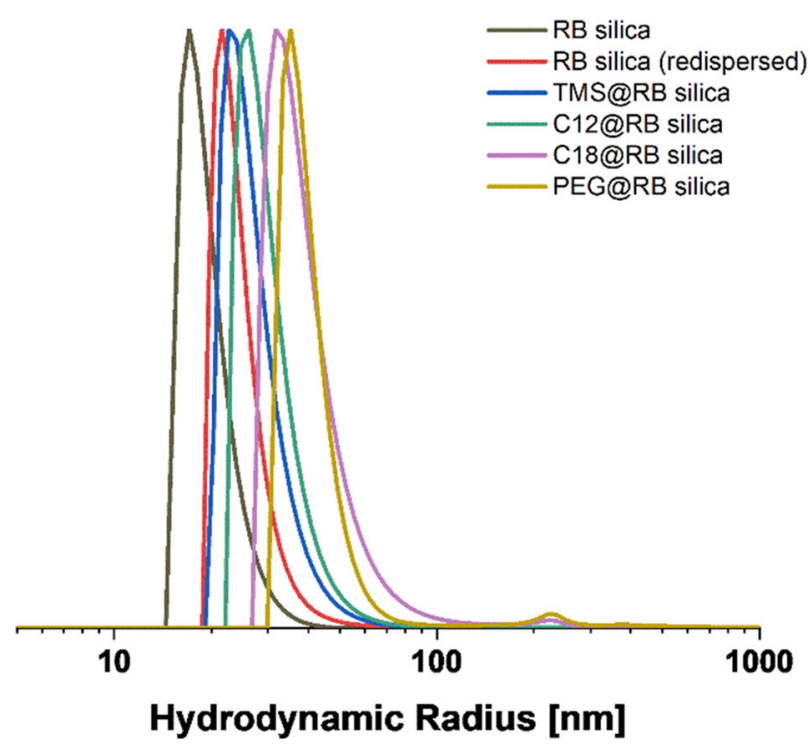

Fig. 8 DLS analysis of rhodamine B silica and the surface alkylated particles; all measurements were carried out in ethanol 


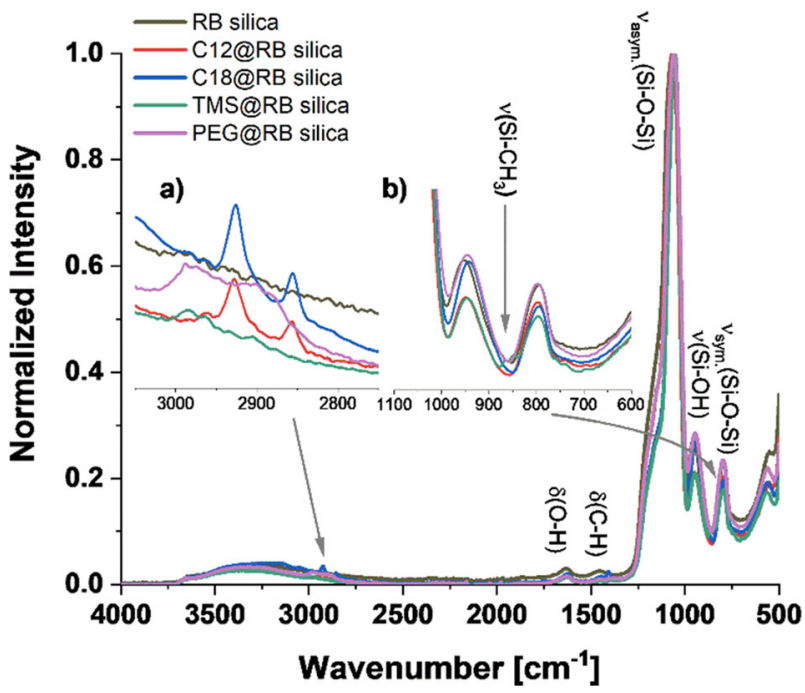

Fig. 9 . FT-IR spectra of rhodamine B silica NPs and different alkyl functionalized rhodamine B NPs; a shows alkyl modes of C12 and C18 functionalized silica, $\mathbf{b}$ TMS functionalization can be seen via the $\mathrm{Si}-\mathrm{CH}_{3}$ mode at $848 \mathrm{~cm}^{-1}$

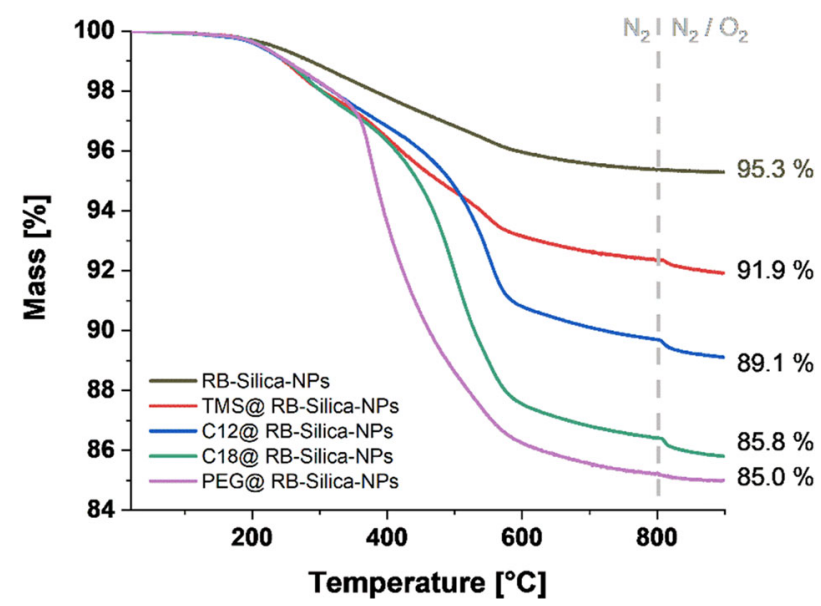

Fig. 10 TGA of alkyl and PEG functionalized RB silica NPs

the functionalized particles decrease as expected with increasing chain length. TMS functionalization shows a residual mass of $92.1 \%$, dodecyl-functionalization of $89.1 \%$, and with octadecyl-functionalization of $85.8 \%$.

The degree of functionalization can be calculated from the TGA residual mass and the carbon content from the CHN analysis, taking into account the molar mass $\mathrm{M}_{\mathrm{C}}$ and number of contributing carbon atoms $\mathrm{N}$ of the functionalization (Eq. (2)) [14]. $\Delta \mathrm{m}_{\mathrm{SiO} 2}$ is the TGA residual mass, $\mathrm{M}_{\mathrm{C}}$ the molar mass of carbon and $\mathrm{N}$ the number of carbon atoms of the functionalization agent.

$c_{F G}=\frac{100}{\Delta m_{S_{i O}}} \times \frac{\Delta m_{C}}{M_{C}} \times \frac{0.01}{N}$
$\Delta m_{C}=C[\%]_{\text {funct } R B-\text { silica }}-C[\%]_{R B-\text { silica }}$

Since some unhydrolyzed ethoxyl groups or ethanol solvent molecules are also present in the unfunctionalized particles, their carbon content must be subtracted from that of the functionalized particles to obtain only the contribution of the functionalization $\Delta \mathrm{m}_{\mathrm{C}}$ (Eq. (3)). In line with expectations, the carbon content increases with increasing molar mass of the functionalizing reagent from $1.28 \%$ of unfunctionalized dye doped particles up to $7.37 \%$ for particles with $\mathrm{C} 18$ shell (Table 2). We reached a total mass loss of $11.0 \%$ for C18@RB-silica without subtraction of the mass loss caused by the drying step, which is comparable to the $10.5 \%$ reached by Sriramulu et al. [5]. Based on the used $0.40 \mathrm{mmol}$ per gram particle functionalization reagent, good conversions of 78 to $88 \%$ can be achieved with this method.

In addition, the covalent attachment of the alkyl groups to the particles can be detected by means of CP/MAS measurements. In the ${ }^{13} \mathrm{C}-\mathrm{CP} / \mathrm{MAS}$ of pure RB-silica particles, only the characteristic signals of ethoxy groups $\left(\mathrm{CH}_{3}\right.$ : $16.68 \mathrm{ppm}$ and $\mathrm{CH}_{2}: 58.57 \mathrm{ppm}$ ), which originate from incompletely hydrolyzed TEOS groups and from solvent residues, can be seen (Fig. S1). The TMS signals are at $-1.29 \mathrm{ppm}$. The alkyl signals of the $\mathrm{C}_{12}$ and $\mathrm{C}_{18}$ functionalization are located at $12.19 \mathrm{ppm}, 22.06 \mathrm{ppm}, 29.09 \mathrm{ppm}$, $31.62 \mathrm{ppm}$, and $58.57 \mathrm{ppm}$.

The bonding mode of the surface functionalization of silica can be detected by ${ }^{29} \mathrm{Si}-\mathrm{CP} / \mathrm{MAS}$ (Fig. 2). In principle, the alkylsilanes can link with the silica surface via a maximum of three bonds. If this occurs, $\mathrm{T}^{3}$ signals can be seen. When bound by only two or one oxo bonds, $\mathrm{T}^{2}$ and $\mathrm{T}^{1}$ signals, respectively, can be observed. In the prepared particles with $\mathrm{C}_{12}$ and $\mathrm{C}_{18}$ groups, $\mathrm{T}^{3}$ and $\mathrm{T}^{2}$ signals are found at $-66.31 \mathrm{ppm}$ and $-56.91 \mathrm{ppm}$. Since neither $\mathrm{T}^{1}$ nor $\mathrm{T}^{0}$ appears, there is no longer free functionalizing agent. Signals of RB-silica are assigned to $\mathrm{Q}^{4}$ at $-110.14, \mathrm{Q}^{3}$ at $-101.87 \mathrm{ppm}$ and $\mathrm{Q}^{2}$ at $-92.53 \mathrm{ppm}$. TMS functionalization can be assigned as small $\mathrm{M}^{1}$ signal at $12.99 \mathrm{ppm}$.

All solid-state NMR signals of the surface functionalizations are listed in Table S2.

\subsection{Leaching process}

\subsubsection{Particle separation}

Physically bound dyes tend to be washed out of the particles over time, while covalent binding of the dye largely prevents this process [11], although the exact nature of the latter has not been described in detail. To get an overview concerning differences between the nature of dye 
Table 2 Residual masses from TGA measurements, carbon content from $\mathrm{CHN}$-analysis and calculated concentration of functionalization groups on the particle surfaces

\begin{tabular}{lllllll}
\hline Particle & $\Delta m_{\mathrm{SiO}_{2}} \cdot[\%]$ & $\mathrm{C}[\%]$ & $\Delta m_{c}[\%]$ & $N$ & $\mathrm{c}_{\mathrm{FG}}[\mathrm{mmol} / \mathrm{g}]$ & Conversion [\%] \\
\hline RB-Silica & 95.3 & 1.28 & 0 & 0 & 0 & - \\
TMS@RB-Silica & 91.9 & 2.32 & 1.04 & 3 & 0.31 & 78 \\
C12@RB-Silica & 89.1 & 5.80 & 4.52 & 12 & 0.35 & 88 \\
C18@RB-Silica & 85.8 & 7.37 & 6.09 & 18 & 0.33 & 83 \\
PEG@RB-Silica & 85.0 & 5.86 & 10.30 & $16-22$ & $0.46-0.63$ & $58-79$ \\
\hline
\end{tabular}

Scheme 6 Stepwise leaching experiment. The washed particles were diluted in ethanol and were stirred for at least 24 hours. Then the particles were separated and measurement of the remaining fluorescence in the centrifugate was measured

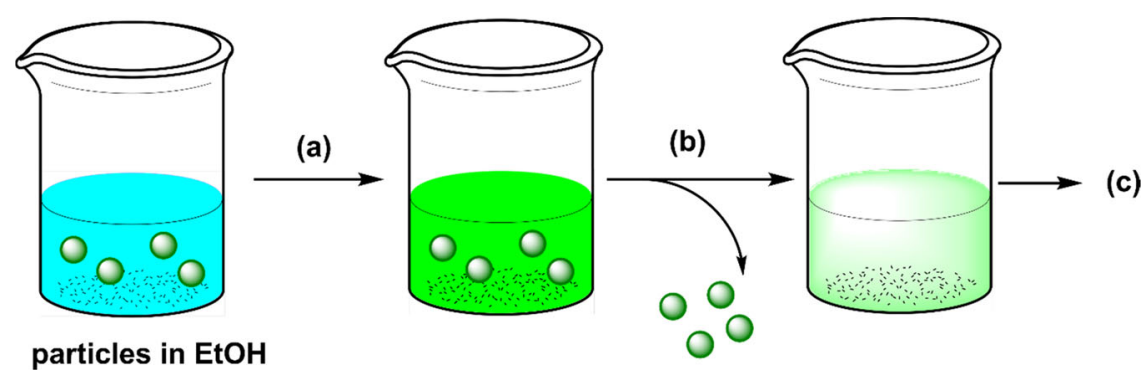

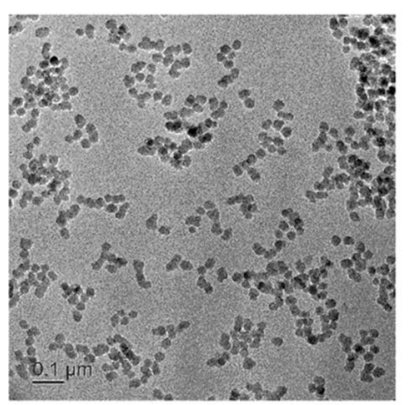

(a)

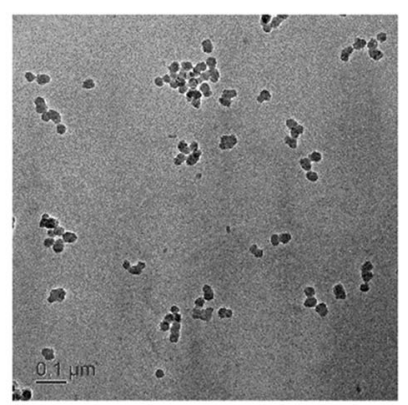

(b)

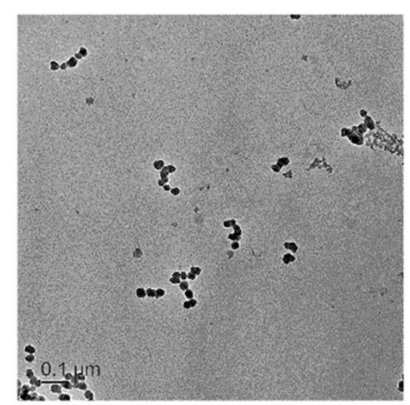

(c)

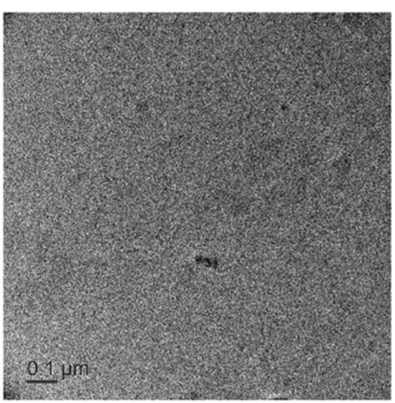

(d)

Fig. 11 TEM images of particle removal process. a Particle dispersion before leaching b Removal of particles after centrifugation $\mathbf{c}$ Removal of most particles after centrifugation and filtration $\mathbf{d}$ filtrate after separation with $10 \mathrm{kDa}$ membrane

connection, rhodamine B and uranine were applied in their salt forms and with anchor groups.

Two types of leaching experiments were performed: the core particles with covalently bound and adsorbed dye were used, and then a leaching of the core particles with surface functionalization (which hinders the leaching of the adsorbed dyes) was carried out.

The leaching study was carried out with diluted stock solutions. The particle dispersion prepared was used as a measure of maximum absorption. Then, the particle dispersions were stirred in ethanol for up to four days. During the stirring time, weakly attached dye molecules will leach out in the solvent. Every day, a small aliquot was removed, and the solvent was separated from the particles (Scheme 6). From all particle dispersions, triplicates were created to ensure reproducibility.

A complete removal of small nanoparticles below $200 \mathrm{~nm}$ is difficult since centrifugation and syringe filtration are not sufficient to remove all particles, and hence several separation steps have been performed. First, the dispersion was centrifuged, with many particles already settling. The centrifugates were then filtered with $0.2 \mu \mathrm{m}$ syringe filters. After these two steps, about $75 \%$ of all particles had already been removed, which can be proven by TEM images (Fig. 11). Centrifuge tubes with $10 \mathrm{kDa}$ membranes can be used for complete particle separation. These were only applied in the last leaching step to show comparability of the samples. Since the separation process was the same for all samples, the extent of the remaining particles in all samples is about the same and thus can be compared.

\subsubsection{Scattering correction of UV/Vis measurements}

Through measurement with an UV/Vis device with an integrating sphere, scattering effects of the particles were minimized. In order to remove the remaining scattering effects from the measurement which affect the detected intensity, a baseline correction was made [38]. The detected absorption follows the Lambert-Beer law (4), where I and $\mathrm{I}_{0}$ 
are the detected and incoming light intensity and $\mathrm{A}_{0}$ the baseline shift in the y-direction.

$A=\log \frac{I_{0}}{I}+A_{0}$

Particles which are smaller than the wavelength of the irradiated light produce Rayleigh scattering. This scattered light is lost during measurements with conventional UV/Vis devices and is subtracted from the detected light intensity,

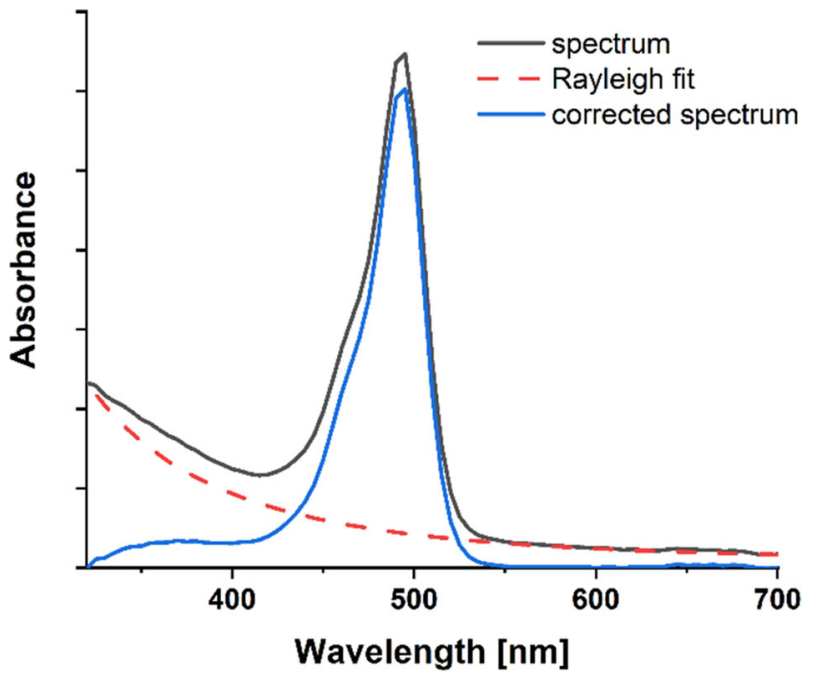

Fig. 12 Example for baseline correction of a scattering sample

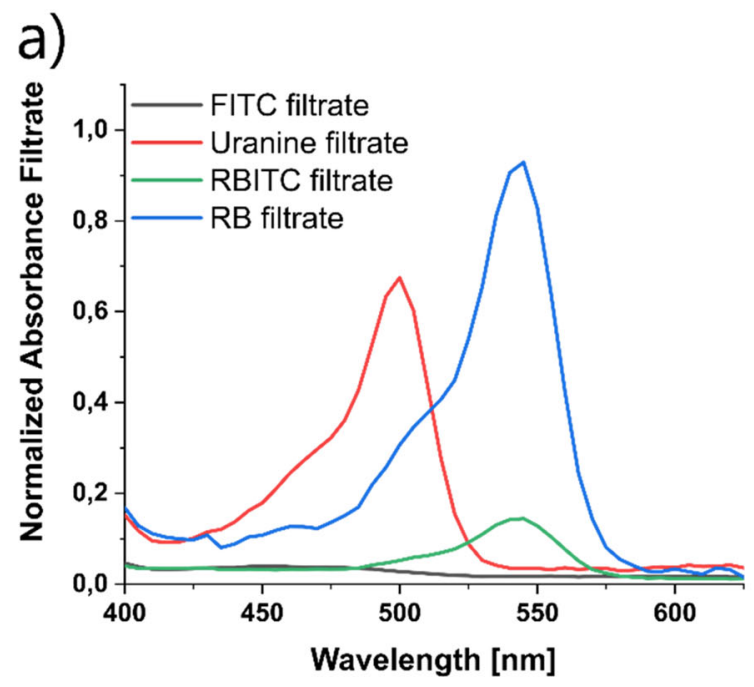

which results in a modified form of the Lambert-Beer law (5).

$A=\log \frac{I_{0}}{I_{0}-I_{\text {scatter }}}+A_{0}$

This effect is the strongest at small wavelengths due to the $\mathrm{I}^{-4}$ dependency of Rayleigh scattering. Using a constant of proportionality $\mathrm{c}$ and the $\mathrm{I}^{-4}$ dependency, Eq. (5) transforms to $(\mathbf{6})$.

$$
A=\log \frac{1}{1-c \times \lambda^{-4}}+A_{0}
$$

From a measured absorption spectrum of a particle dispersion, the Rayleigh fraction can now be subtracted (Fig. 12).

\subsubsection{Leaching results}

As expected, the particles with physisorbed dye (rhodamine $\mathrm{B}$ and uranine) had already leached out a large part of the dye after the first day (Fig. 13). This effect is even greater for rhodamine B, which shows an absorbance in the centrifugate of 0.98 after one day of leaching compared to that for uranine, whose absorbance is 0.54. After four days, rhodamine B was essentially completely leached out (absorbance 0.99) and uranine leaching was nearly complete (absorbance 0.93 ). The fact that the covalently bonded dyes show a measurable absorption of the filtrate at the beginning is due to a few remaining particles that could not

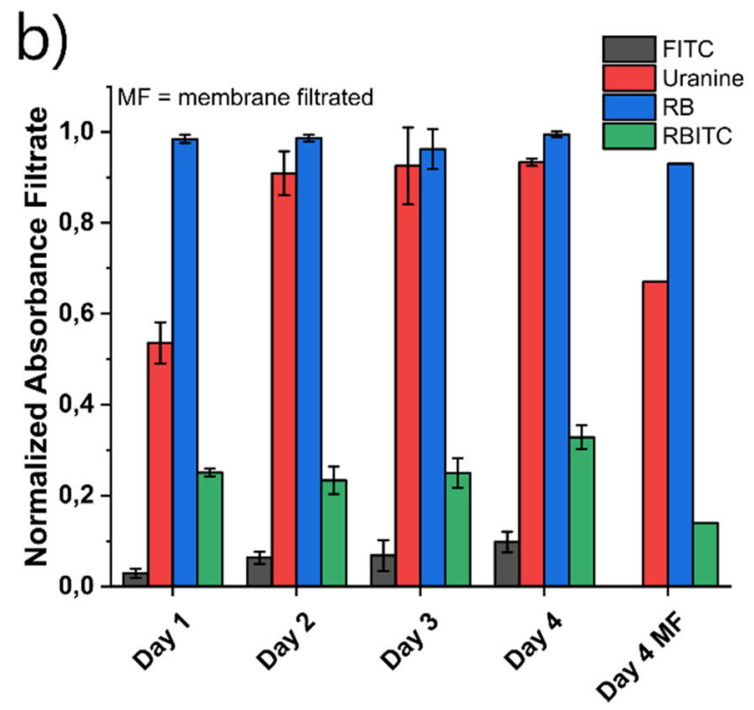

Fig. 13 a Absorbance spectra of the particle-free filtrates after four days leaching. The spectra are normalized to the spectra of the baseline corrected particle dispersions. b Overview of the leaching behavior of different dye labeled particles. Leaching was performed in ethanol at room temperature; all samples were investigated three times. Non-covalently attached dyes (rhodamine B (RB), uranine) tend to leach nearly complete out after the first two leaching steps, covalently bonded dyes (RBITC and FITC connected via APTES to the particle) show only a small tendency to leach. Samples were taken after 1 day, 2 days, 3 days, and 4 days ( $24 \mathrm{~h}$ intervals). After day four, the sample was also filtered with a $10 \mathrm{kDa}$ membrane filter (MF) for complete particle removal 
be removed by centrifugation and filtration (as shown in Fig. 11).

In contrast to FITC-containing particles, RBITC core particles also show a dye loss after membrane filtration. In the case of RBITC-core particles, it would be possible that after the particle synthesis a few free molecules remain which are adsorbed, for example, in deeper pores of the particles and are dissolved out by the solvent only after a long time. After one day, absorbances of 0.03 for FITC and of 0.25 for RBITC can be found in the filtrate; the corresponding values after 4 days are 0.1 for FITC and 0.33 for RBITC. The overall increase in absorbance for both dyes is comparable ( 0.07 for FITC and 0.08 for RBITC), indicating similar processes of dye loss.

Both as a physisorbed dye and in its covalently bonded form, rhodamine B seems to be more prone to leaching than the fluorescein dyes. This effect seems to have nothing to do with solubility, since the solubility of rhodamine B in ethanol is even slightly lower than that of fluorescein (solubility in ethanol of RBITC: $15 \mathrm{mg} / \mathrm{mL}$ [53], FITC:

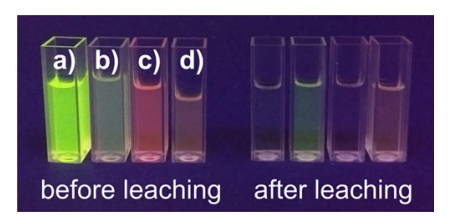

Fig. 14 Fluorescence of particle- and dye solutions under UV light. Left: particle dispersions in ethanol; right: particle free filtrates after a four-day leaching. Dye labels: a FITC, b uranine, c RBITC, d rhodamine B. Particles with covalently attached dyes show nearly no visible dye loss while filtrates of particles with physisorbed dyes are strongly colored

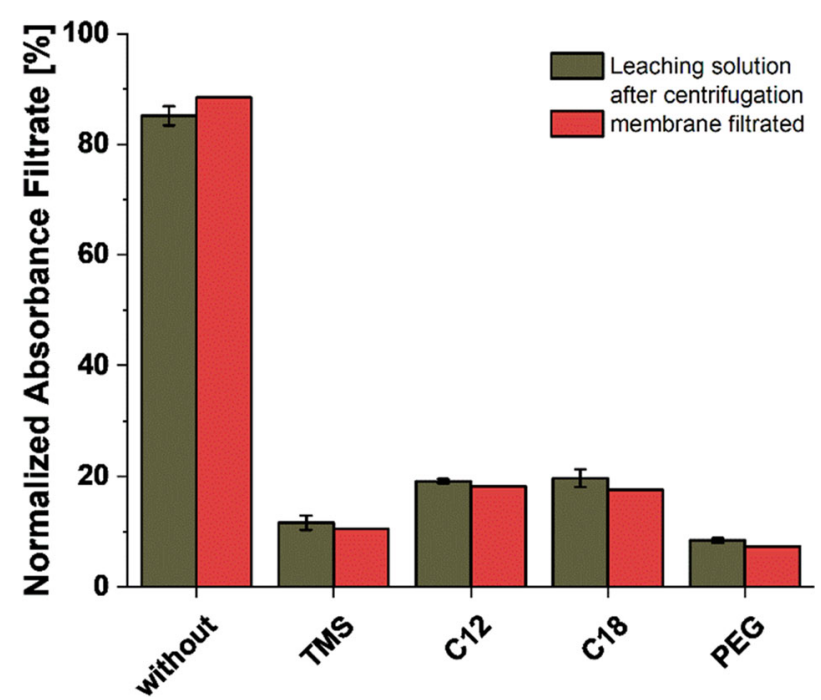

Fig. 15 Absorbance of leaching filtrates of particles with esterified rhodamine B after $24 \mathrm{~h}$. Leaching behavior of unfunctionalized RB particles with esterified dye is slightly better than without ester bond; an alkyl or PEG functionalization improves the dye retention
$20 \mathrm{mg} / \mathrm{mL}$ [54]). It would be conceivable that rhodamine B has a slightly higher steric demand due to the diethyl amine groups instead of smaller hydroxy groups and for this reason is less efficiently incorporated into the silica matrix than FITC.

After the membrane filtration, the filtrates of the rhodamine B and uranine particles still show a strong color. No visually discernible coloration can be observed in the filtrate of the FITC-modified particles, while the filtrate of the RBITC-modified particles is slightly colored (Fig. 14).

The obtained absorbance spectra after membrane filtration show the same trend as seen before: a high absorbance for the physisorbed dyes and rather low ones for the covalently attached dyes (Fig. 13a). As expected, all intensities after membrane filtration are lower compared to the filtrates without the membrane filtration step, due to the complete particle removal (Fig. 13b). For FITC-NPs, even no signal can be detected. This means that the detected absorbance before membrane filtration does not arise from leached molecular dye, but from particles.

The leaching of the physisorbed dyes should be improved by surface functionalization with organic groups. The alkylated and the PEG-modified particles were analyzed for their leaching behavior in the same way as for the core particles. After stirring for $24 \mathrm{~h}$, samples were taken. The esterification of rhodamine $\mathrm{B}$ leads to a slightly reduced leaching of the particles without protective shell in comparison to particles with adsorbed rhodamine B (85\% instead of $99 \%$, Figs. 13b and 15).

By alkylation leaching is further reduced. Longer alkyl chains show a similar leaching of $19 \%$ for $\mathrm{C} 12$ and $\mathrm{C} 18$; the TMS functionalization is slightly more effective with $12 \%$. PEG functionalization is the most effective, with only $8 \%$ dye found in the filtrate (Fig. 15). For Stöber particles, an increase in the interaction between particle and adsorbed dye thus seems to be necessary. This can be achieved for example with $\pi-\pi$ interaction between particle matrix and dye [10] or by adding a silica shell [20,55]. The esterification of the xanthene dyes without anchor group offers a simple alternative to prevent dye leaching. Through subsequent surface functionalization, the dye retention further increases and additionally, the particle dispersibility in hydrophobic and hydrophilic media can be optimized. The PEG functionalization turned out to be most effective to prevent dye loss. Due to the high steric demand of the PEG groups, the dye molecules are effectively retained. This effect is slightly weaker for the shorter alkyl chains (C12 and C18), and more dye is lost. The TMS functionalization also ensures good dye retention, which can be explained by the slightly higher density of functionalization, since three methyl groups are applied to the silica surface per TMS molecule. 


\section{Conclusion}

Different xanthene dyes (rhodamine B, rhodamine B isothiocyanate, uranine, fluorescein isothiocyanate) were incorporated into silica nanoparticles via a modified Stöber synthesis. It was found that the binding mode of the dye has a significant influence on the stability of the fluorescence of the particles. Particles with covalently bound dyes show only a very weak leaching behavior, which is probably due to adsorbed dye molecules which were not completely removed by the washing steps. Physically bound dyes show in general a strong leaching; the dyes leach nearly completely out of the particles after a few washing steps. Rhodamine B and its derivative RBITC show a stronger tendency to be washed out than the fluorescein derivatives uranine and FITC, which might be due to steric effects. To improve the retention of physically bound dyes, core-shell particles were produced. The different lengths of alkyl chains create a barrier that locks the dye molecules sterically and through their lipophilicity into the particle. Pure adsorption on the particles does not prevent the xanthene molecules from leaching. It is necessary to intensify the interaction between dye and particle. This can be achieved in a relatively simple way by a preliminary esterification of the dye. By building a coreshell structure with either hydrophobic or hydrophilic groups, leaching can be further reduced, and, in addition, the particles can be adapted to the desired requirements. The leaching experiments show that the type and the stability of dye incorporation is an important factor for all applications that use labeled silica particles.

Acknowledgements We thank Dr. Michael Zimmer for the CP/MAS spectra, as well as Dr. Anne Leutzgen for fluorescence anisotropy measurements and helpful discussions. In addition, we thank Susanne Harling for the CHN analysis.

Author contributions The manuscript was written through contributions of all authors. All authors have given approval to the final version of the manuscript.

Funding Open Access funding enabled and organized by Projekt DEAL.

\section{Compliance with ethical standards}

Conflict of interest The authors declare no competing interests.

Publisher's note Springer Nature remains neutral with regard to jurisdictional claims in published maps and institutional affiliations.

Open Access This article is licensed under a Creative Commons Attribution 4.0 International License, which permits use, sharing, adaptation, distribution and reproduction in any medium or format, as long as you give appropriate credit to the original author(s) and the source, provide a link to the Creative Commons license, and indicate if changes were made. The images or other third party material in this article are included in the article's Creative Commons license, unless indicated otherwise in a credit line to the material. If material is not included in the article's Creative Commons license and your intended use is not permitted by statutory regulation or exceeds the permitted use, you will need to obtain permission directly from the copyright holder. To view a copy of this license, visit http://creativecommons. org/licenses/by/4.0/.

\section{References}

1. Aslan K, Wu M, Lakowicz JR, Geddes CD (2007) Fluorescent core-shell Ag@SiO2 nanocomposites for metal-enhanced fluorescence and single nanoparticle sensing platforms. J Am Chem Soc 129:1524-1525. https://doi.org/10.1021/ja0680820

2. Hasegawa U, Nomura SIM, Kaul SC et al. (2005) Nanogelquantum dot hybrid nanoparticles for live cell imaging. Biochem Biophys Res Commun 331:917-921. https://doi.org/10.1016/j. bbrc.2005.03.228

3. Shirshahi V, Soltani M (2015) Solid silica nanoparticles: applications in molecular imaging. Contrast Media Mol Imaging 10:1-17. https://doi.org/10.1002/cmmi.1611

4. Jung H-S, Kim Y-J, Ha S-W, Lee J-K (2013) White light-emitting diodes using thermally and photochemically stable fluorescent silica nanoparticles as color-converters. J Mater Chem C 1:5879-5884. https://doi.org/10.1039/c3tc30967c

5. Gupta S, Zhang Q, Emrick T et al. (2006) Entropy-driven segregation of nanoparticles to cracks in multilayered composite polymer structures. Nat Mater 5:229-233. https://doi.org/10.1038/ nmat 1582

6. Stöber W, Fink A, Bohn E (1968) Controlled growth of monodisperse silica spheres in the micron size range. J Colloid Interface Sci 26:62-69. https://doi.org/10.1016/0021-9797(68)90272-5

7. Arriagada FJ, Osseo-Assare K (1995) Synthesis of nanosize silica in aerosol OT reverse microemulsions. J Colloid Interface Sci 170:8-17. https://doi.org/10.1006/jcis.1995.1064

8. Hartlen KD, Athanasopoulos APT, Kitaev V (2008) Facile preparation of highly monodisperse small silica spheres $(15$ to $>200$ $\mathrm{nm}$ ) suitable for colloidal templating and formation of ordered arrays. Langmuir 24:1714-1720. https://doi.org/10.1021/la 7025285

9. Shahabi S, Treccani L, Rezwan K (2015) Amino acid-catalyzed seed regrowth synthesis of photostable high fluorescent silica nanoparticles with tunable sizes for intracellular studies. J Nanoparticle Res 17:270. https://doi.org/10.1007/s11051-015-3072-8

10. Tapec R, Zhao XJ, Tan W (2002) Development of organic dyedoped silica nanoparticles for bioanalysis and biosensors. J Nanosci Nanotechnol 2:405-409

11. Tolbert SH, Loy DA (2015) Fluorescent hybrid organic - inorganic particles: in fluence of physical encapsulation versus covalent attachment on leaching and UV stability. J Ceram Soc Japan 123:785-792. https://doi.org/10.2109/jcersj2.123.785

12. Hammond VJ, Aylott JW, Greenway GM et al. (2008) An optical sensor for reactive oxygen species: encapsulation of functionalised silica nanoparticles into silicate nanoprobes to reduce fluorophore leaching. Analyst 133:71-75. https://doi.org/10.1039/b711995j

13. Schneider M, Müllen K (2000) Hybrid materials doped with covalently bound perylene dyes through the sol-gel process. Chem Mater 12:352-362. https://doi.org/10.1021/cm9910613

14. van Blaaderen A, Vrij A (1992) Synthesis and characterization of colloidal dispersions of fluorescent, monodisperse silica spheres. Langmuir 8:2921-2931. https://doi.org/10.1021/la00048a013

15. Verhaegh NAM, van Blaaderen A (1994) Dispersions of rhodamine-labeled silica spheres: synthesis, characterization, and fluorescence confocal scanning laser microscopy. Langmuir 10:1427-1438. https://doi.org/10.1021/la00017a019 
16. Zhang L, Veerapandian M, Yun KS (2014) Fluorescent silica nanoparticles functionalized on multi-walled carbon nanotubes: Fabrication and fluorescent properties. BioChip J 8:83-90. https:// doi.org/10.1007/s13206-014-8203-2

17. Santra S, Liesenfeld B, Bertolino C et al. (2006) Fluorescence lifetime measurements to determine the core-shell nanostructure of FITC-doped silica nanoparticles: an optical approach to evaluate nanoparticle photostability. J Lumin 117:75-82. https://doi.org/ 10.1016/j.jlumin.2005.04.008

18. Mader H, Li X, Saleh S et al. (2008) Fluorescent silica nanoparticles. Ann NY Acad Sci 1130:218-223. https://doi.org/10. 1196/annals. 1430.053

19. Ribeiro T, Baleizão C, Farinha JPS (2009) Synthesis and characterization of perylenediimide labeled core - shell hybrid silica polymer nanoparticles. J Phys Chem C 113:18082-18090

20. Ethiraj AS, Hebalkar N, Kharrazi S et al. (2005) Photoluminescent core-shell particles of organic dye in silica. J Lumin 114:15-23. https://doi.org/10.1016/j.jlumin.2004.11.005

21. Zane A, McCracken C, Knight DA et al. (2015) Uptake of bright fluorophore core-silica shell nanoparticles by biological systems. Int J Nanomedicine 10:1547-1567. https://doi.org/10.2147/IJN.S76208

22. Burns A, Ow H, Wiesner U (2006) Fluorescent core-shell silica nanoparticles: towards "Lab on a Particle" architectures for nanobiotechnology. Chem Soc Rev 35:1028-1042. https://doi. org/10.1039/b600562b

23. Burns A, Sengupta P, Zedayko T et al. (2006) Core/shell fluorescent silica nanoparticles for chemical sensing: Towards singleparticle laboratories. Small 2:723-726. https://doi.org/10.1002/ smll.200600017

24. Fuller JE, Zugates GT, Ferreira LS et al. (2008) Intracellular delivery of core-shell fluorescent silica nanoparticles. Biomaterials 29:1526-1532. https://doi.org/10.1016/j.biomaterials.2007.11.025

25. Herz E, Burns A, Lee S et al. (2006) Fluorescent core-shell silica nanoparticles: an alternative radiative materials platform. SPIE 6096:609605. https://doi.org/10.1117/12.661782

26. Larson DR, Ow H, Vishwasrao HD et al. (2008) Silica nanoparticle architecture determines radiative properties of encapsulated fluorophores silica nanoparticle architecture determines radiative properties of encapsulated fluorophores. Chem Mater 20:2677-2684. https://doi.org/10.1021/cm7026866

27. Yang H-H, Qu H-Y, Lin P et al. (2003) Nanometer fluorescent hybrid silica particle as ultrasensitive and photostable biological labels. Analyst 128:462-466. https://doi.org/10.1039/b210192k

28. Wang H, Schaefer K, Pich A, Moeller M (2011) Synthesis of silica encapsulated perylenetetracarboxylic diimide core-shell nanoellipsoids. Chem Mater 23:4748-4755. https://doi.org/10. $1021 / \mathrm{cm} 2017328$

29. Zhao X, Bagwe RP, Tan W (2004) Development of organic-dyedoped silica nanoparticles in a reverse microemulsion. Adv Mater 16:173-176. https://doi.org/10.1002/adma.200305622

30. Stylianopoulos T (2013) EPR-effect: utilizing size-dependent nanoparticle delivery to solid tumors. Ther Deliv 4:421-423. https://doi.org/10.4155/tde.13.8

31. Kim J, Kim HS, Lee $\mathrm{N}$ et al. (2008) Multifunctional uniform nanoparticles composed of a magnetite nanocrystal core and a mesoporous silica shell for magnetic resonance and fluorescence imaging and for drug delivery. Angew Chemie - Int Ed 47:8438-8441. https://doi.org/10.1002/anie.200802469

32. Nyffenegger R, Quellet C, Ricka J (1993) Synthesis of Fluorescent, Monodisperse, Colloidal Silica Particles. J Colloid Interface Sci 159:150-157. https://doi.org/10.1006/jcis.1993.1306

33. Han Y, Lu Z, Teng $\mathrm{Z}$ et al. (2017) Unraveling the growth mechanism of silica particles in the stöber method: in situ seeded growth model. Langmuir 33:5879-5890. https://doi.org/10.1021/a cs.langmuir.7b01140
34. Weber G (1960) Fluorescence-polarization spectrum and electronicenergy transfer in tyrosine, tryptophan and related compounds. Biochem J 75:335-345. https://doi.org/10.1042/bj0750335

35. Ghosh S, Saha S, Goswami D, et al (2012) Dynamic imaging of homo-FRET in live cells by fluorescence anisotropy microscopy, 1st ed. Elsevier Inc

36. Hsu C-Y, Liu Y-L (2010) Rhodamine B-anchored silica nanoparticles displaying white-light photoluminescence and their uses in preparations of photoluminescent polymeric films and nanofibers. J Colloid Interface Sci 350:75-82. https://doi.org/10.1016/j. jcis.2010.06.011

37. Sriramulu D, Reed EL, Annamalai M et al. (2016) Synthesis and Characterization of Superhydrophobic, Self-cleaning NIR-reflective Silica Nanoparticles. Sci Rep 6:35993. https://doi.org/10. 1038/srep35993

38. Preus S (2015) Scatter in UV-Vis absorption spectra. http://www. fluortools.com/software/ae/documentation/tools/scatter. Accessed 11 Oct 2018

39. Imhof A, Megens M, Engelberts JJ et al. (1999) Spectroscopy of Fluorescein (FITC) Dyed Colloidal Silica Spheres. J Phys Chem B 103:1408-1415. https://doi.org/10.1021/jp983241q

40. Bogush GH, Tracy MA, Zukoski CFI (1988) Preparation of monodisperse silica particles: control of size and mass fraction. J Non Cryst Solids 104:95-106

41. Pabisch S, Feichtenschlager B, Kickelbick G, Peterlik H (2012) Effect of interparticle interactions on size determination of zirconia and silica based systems-A comparison of SAXS, DLS, BET, XRD and TEM. Chem Phys Lett 521:91-97. https://doi.org/ 10.1016/j.cplett.2011.11.049

42. Tavernaro I, Cavelius C, Peuschel H, Kraegeloh A (2017) Bright fluorescent silica-nanoparticle probes for high-resolution STED and confocal microscopy. Beilstein J Nanotechnol 8:1283-1296. https://doi.org/10.3762/bjnano.8.130

43. Quignard S, Mosser G, Boissière M, Coradin T (2012) Long-term fate of silica nanoparticles interacting with human dermal fibroblasts. Biomaterials 33:4431-4442. https://doi.org/10.1016/j. biomaterials.2012.03.004

44. Montalti M, Prodi L, Zaccheroni N et al. (2006) Size effect on the fluorescence properties of dansyl-doped silica nanoparticles. Langmuir 22:5877-5881. https://doi.org/10.1021/la053473y

45. Mchedlov-Petrossyan NO, Kukhtik VI, Bezugliy VD (2003) Dissociation, tautomerism and electroreduction of xanthene and sulfonephthalein dyes in N,N-dimethylformamide and other solvents. J Phys Org Chem 16:380-397. https://doi.org/10.1002/ poc. 654

46. Batistela VR, Pellosi DS, De Souza FD et al. (2011) PKa determinations of xanthene derivates in aqueous solutions by multivariate analysis applied to $\mathrm{UV}-\mathrm{Vis}$ spectrophotometric data. Spectrochim Acta - Part A Mol Biomol Spectrosc 79:889-897. https://doi.org/10.1016/j.saa.2011.03.027

47. Clasen A, Wenderoth S, Tavernaro I et al. (2019) Kinetic and spectroscopic responses of $\mathrm{pH}$-sensitive nanoparticles: Influence of the silica matrix. RSC Adv 9:35695-35705. https://doi.org/10. 1039/c9ra06047b

48. Jameson DM, Croney JC (2003) Fluorescence polarization: past, present and future. Comb Chem High Throughput Screen 6:167-176

49. Valeur B, Brochon J-C (2001) New Trends in Fluorescence Spectroscopy. Springer-Verlag Berlin Heidelberg

50. Lakowicz JR (2006) Principles of Fluorescence Spectroscopy, Third Edn. Springer, Baltimore, Maryland, USA

51. Blechinger J, Herrmann R, Kiener D et al. (2010) Perylene-labeled silica nanoparticles: Synthesis and characterization of three novel silica nanoparticle species for live-cell imaging. Small 6:2427-2435. https://doi.org/10.1002/smll.201000762 
52. Hsu C-Y, Liu Y-L (2010) Rhodamine B-anchored silica nanoparticles displaying white-light photoluminescence and their uses in preparations of photoluminescent polymeric films and nanofibers. J Colloid Interface Sci 350:75-82. https://doi.org/10.1016/j. jcis.2010.06.011

53. (2017) RBITC Product Information. https://www.caymanchem. com/pdfs/20653.pdf. Accessed 11 Oct 2018
54. FITC Product Information. https://www.sigmaaldrich.com/ content/dam/sigma-aldrich/docs/Sigma/Product_Information Sheet/f7250pis.pdf. Accessed 11 Oct 2018

55. Daberkow T, Meder F, Treccani L et al. (2012) Fluorescence labeling of colloidal core-shell particles with defined isoelectric points for in vitro studies. Acta Biomater 8:720-727. https://doi. org/10.1016/j.actbio.2011.11.007 